\title{
Field quantization in inhomogeneous absorptive dielectrics
}

\author{
L. G. Suttorp ${ }^{1}$ and Martijn Wubs ${ }^{2,3}$ \\ ${ }^{1}$ Institute for Theoretical Physics, University of Amsterdam, Valckenierstraat 65, NL-1018 XE Amsterdam, The Netherlands \\ ${ }^{2}$ Complex Photonic Systems, Faculty of Science and Technology, University of Twente, P. O. Box 217 , \\ NL-7500 AE Enschede, The Netherlands \\ ${ }^{3}$ Van der Waals-Zeeman Institute, University of Amsterdam, Valckenierstraat 65, NL-1018 XE Amsterdam, The Netherlands
}

(Received 2 March 2004; published 30 July 2004)

\begin{abstract}
The quantization of the electromagnetic field in a three-dimensional inhomogeneous dielectric medium with losses is carried out in the framework of a damped-polariton model with an arbitrary spatial dependence of its parameters. The equations of motion for the canonical variables are solved explicitly by means of Laplace transformations for both positive and negative time. The dielectric susceptibility and the quantum noise-current density are identified in terms of the dynamical variables and parameters of the model. The operators that diagonalize the Hamiltonian are found as linear combinations of the canonical variables, with coefficients depending on the electric susceptibility and the dielectric Green function. The complete time dependence of the electromagnetic field and of the dielectric polarization is determined. Our results provide a microscopic justification of the phenomenological quantization scheme for the electromagnetic field in inhomogeneous dielectrics.
\end{abstract}

DOI: 10.1103/PhysRevA.70.013816

PACS number(s): 42.50.Nn, 42.50.-p, 41.20.Jb

\section{INTRODUCTION}

The quantization of the electromagnetic field in a linear dielectric medium has been the subject of many investigations since the first treatment by Jauch and Watson [1]. In a homogeneous and nondispersive medium the photon is associated with the transverse part of the field, which can neatly be distinguished from its longitudinal part. In contrast, in an inhomogeneous nondispersive medium the transverse and the longitudinal degrees of freedom get coupled, which renders the generalization of the quantization scheme to that case less straightforward. However, the quantization can still be accomplished by employing a generalized transverse gauge, which depends on the dielectric constant [2-7].

For a dielectric medium with dispersion the quantization procedure described above runs into problems. In fact, since dispersion in a dielectric medium is inextricably connected to extinction, one should account for the effect of losses in the quantization procedure. Huttner and Barnett $[8,9]$ were the first to use the Hopfield polariton model [10] for the description of a dielectric with losses. To incorporate losses they coupled the dielectric polarization to a bath of oscillators, which causes a damping of the polaritons. Subsequently, upon assuming the medium to be homogeneous, they were able to diagonalize the Hamiltonian of this damped-polariton system, and to establish explicit formulas for the electromagnetic field and the dielectric polarization in terms of the diagonalizing operators. As a direct application of the model they evaluated the change in the atomic decay due to a dielectric environment [11]. Later on, their work has been reformulated and extended in various ways. In Refs. $[12,13]$ a simplified expression for the dielectric constant of the model was found. An alternative description of the model in terms of path integrals was given in Ref. [14]. In Ref. [15] Laplace transformations were employed to simplify the diagonalization process considerably. Finally, in Ref. [16] these transforms were used to formulate the equations gov- erning the dynamics of the inhomogeneous model, although a complete diagonalization was not attempted in that case.

As stated above, the treatment in Refs. [8,9] is confined to the homogeneous damped-polariton model. For such systems, a systematic use of spatial Fourier transforms greatly helps in carrying out the diagonalization of the Hamiltonian. Somewhat later it was realized [17-23] that the quantization of the fields in inhomogeneous media could be achieved in position space by adding a noise term to the Maxwell equations in a phenomenological way. In agreement with the fluctuation-dissipation theorem, one then postulates suitable commutation relations for this noise term. Accounting for the Kramers-Kronig relations of the dielectric media is found to be essential in defining field operators with standard commutation relations. An alternative formulation [24] of the quantization procedure by means of auxiliary fields has been shown to be completely equivalent [25].

The phenomenological quantization scheme has been extended to magnetic and to anisotropic media, and to finite media with gain (see the reviews [26,27] and references therein). The scheme has been applied to atomic decay [28-31], to energy transfer [32], and to resonant dipole interactions [33]. The phenomenological scheme has also been used to study the properties of electromagnetic field operators in systems with optical cavities or beam splitters $[34,35]$, and with dielectric slabs [36]. For these optical components input-output relations have been derived. It was found that extinction usually has adverse effects on nonclassical properties of light, such as squeezing, nonclassical correlations and entanglement $[37,38]$.

Although the phenomenological quantization of the electromagnetic field in absorptive dielectrics has been very successful, its connection to the damped-polariton model has been established only for the special case of a homogeneous dielectric medium. In fact, one would like to see whether the crucial properties of the noise term, which are postulated in the phenomenological approach, could be derived from the 
polariton model in the general inhomogeneous case, as has been noted on various occasions $[32,33,39]$. Since the model furnishes a precise Hamiltonian description of the interaction between the damped dielectric and the electromagnetic field, one would have obtained in this way a microscopic justification of the phenomenological quantization scheme. To arrive at this goal, one has to express the noise term in the canonical variables of the model, so that its properties can be determined. Once the noise term has been found, it can be used for the complete diagonalization of the Hamiltonian. In the following we shall show how this can be achieved by employing a Laplace-transform technique as in $[15,16]$.

The paper is organized as follows. In Sec. II the model is defined and the equations of motion for the canonical variables are derived. The Laplace transforms of these are determined in Sec. III. As we shall see, it is essential to introduce forward and backward Laplace transforms for positive and negative time, respectively. Subsequently, in Sec. IV, the Green functions of the inhomogeneous dielectric are employed to determine a space- and frequency-dependent source density, which is the analog of the noise-current density in the phenomenological quantization scheme. The explicit form for this source density as a function of the canonical variables is derived in Sec. V. Once identified in terms of the canonical variables, some important properties of the source density can be derived, as presented in Sec. VI. In particular, we shall demonstrate that the damped-polariton model can be diagonalized in terms of the source density. The full time dependence of the electromagnetic field and of the dielectric polarization density can thus be established. The paper ends with a discussion and with some conclusions.

\section{EQUATIONS OF MOTION}

As a model for an absorptive dielectric interacting with the electromagnetic field we adopt the inhomogeneous damped-polariton system. In this model the polarization density is a continuous space-dependent variable. The damping is provided through the coupling to a bath of harmonic oscillators with a continuous range of eigenfrequencies. The bath coupling constant depends both on the frequency and on the position. The electromagnetic field is coupled to the polarization according to the standard minimal-coupling scheme. [9]

The Lagrangian density of the damped-polariton system is

$$
\begin{aligned}
\mathcal{L}= & \frac{1}{2} \varepsilon_{0} E^{2}-\frac{1}{2} \mu_{0}^{-1} B^{2}+\frac{1}{2} \rho \dot{X}^{2}-\frac{1}{2} \rho \omega_{0}^{2} X^{2}+\frac{1}{2} \rho \int_{0}^{\infty} \mathrm{d} \omega \dot{Y}_{\omega}^{2} \\
& -\frac{1}{2} \rho \int_{0}^{\infty} \mathrm{d} \omega \omega^{2} Y_{\omega}^{2}-\Phi \nabla \cdot(\alpha \mathbf{X})-\alpha \mathbf{A} \cdot \dot{\mathbf{X}} \\
& -\int_{0}^{\infty} \mathrm{d} \omega v_{\omega} \mathbf{X} \cdot \dot{\mathbf{Y}}_{\omega} .
\end{aligned}
$$

The electromagnetic field is described by the scalar potential $\Phi(\mathbf{r})$ and the vector potential $\mathbf{A}(\mathbf{r})$, with $\mathbf{E}=-\nabla \Phi-\dot{\mathbf{A}}$ and $\mathbf{B}=\boldsymbol{\nabla} \times \mathbf{A}$. We choose the Coulomb gauge in which the vec- tor potential is purely transverse, so that $\boldsymbol{\nabla} \cdot \mathbf{A}=0$. The dielectric degrees of freedom are described by a space-dependent harmonic variable $\mathbf{X}(\mathbf{r})$, with an associated eigenfrequency $\omega_{0}(\mathbf{r})$ and a density $\rho(\mathbf{r})$. The polarization density is given by $-\alpha \mathbf{X}$, with $\alpha(\mathbf{r})$ a space-dependent proportionality constant. In the Coulomb gauge the scalar potential is a material variable that is given as the solution of the Poisson equation $\Delta \Phi=-\varepsilon_{0}^{-1} \nabla \cdot(\alpha \mathbf{X})$, with suitable boundary conditions at infinity. The electromagnetic potentials $\Phi, \mathbf{A}$ and the dielectric variable $\mathbf{X}$ are coupled in the usual way, with $\boldsymbol{\nabla} \cdot(\alpha \mathbf{X})$ the bound charge density and $-\alpha \dot{\mathbf{X}}$ the bound current density. Hence, $\alpha(\mathbf{r})$ gives the strength of the coupling between the electromagnetic fields (or the potentials) and $\mathbf{X}(\mathbf{r})$. Damping is introduced in the model through a continuum of harmonicoscillator bath variables $\mathbf{Y}_{\omega}(\mathbf{r})$, labeled by the frequency $\omega$. The coupling to $\mathbf{X}(\mathbf{r})$ is determined by the bath coupling parameter $v_{\omega}(\mathbf{r})$. A schematic representation of the system and its independent parameters is

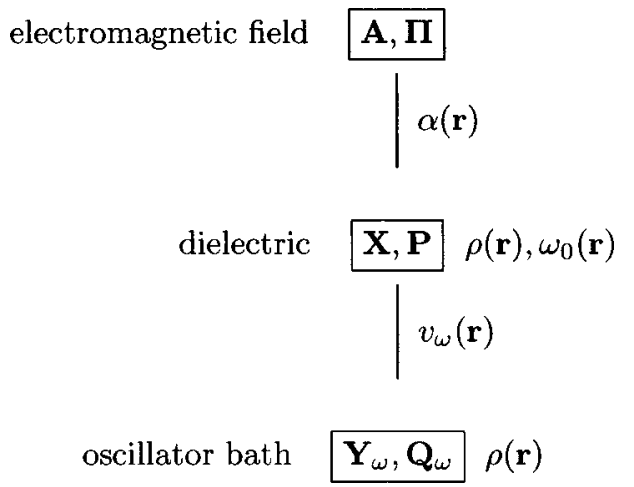

Here, $\boldsymbol{\Pi}, \mathbf{P}$, and $\mathbf{Q}_{\omega}$ are canonical momenta, which will be defined below.

In writing Eq. (1) we have used the same conventions as in Ref. [9]. In particular, we have refrained from a rescaling of the physical variables. As is clear from Eq. (1), the density $\rho$ could have been scaled away by redefining $\mathbf{X}$ and $\mathbf{Y}_{\omega}$. In this way we would have been left with the independent (rescaled) coupling parameters $\alpha$ and $v_{\omega}$ (and the frequency $\omega_{0}$ ). Turning the argument the other way around, we could as well have chosen different density parameters $\rho_{X}$ and $\rho_{Y}$ multiplying the contributions with $\mathbf{X}$ and $\mathbf{Y}_{\omega}$. In that way a model would have been introduced that seems more general, but it is not.

Introducing the canonical momenta

$$
\begin{gathered}
\boldsymbol{\Pi}=\frac{\partial \mathcal{L}}{\partial \dot{\mathbf{A}}}=\varepsilon_{0} \dot{\mathbf{A}}, \\
\mathbf{P}=\frac{\partial \mathcal{L}}{\partial \dot{\mathbf{X}}}=\rho \dot{\mathbf{X}}-\alpha \mathbf{A}, \\
\mathbf{Q}_{\omega}=\frac{\delta \mathcal{L}}{\delta \dot{\mathbf{Y}}_{\omega}}=\rho \dot{\mathbf{Y}}_{\omega}-v_{\omega} \mathbf{X},
\end{gathered}
$$

with $\delta$ a functional derivative in the variable $\omega$, we find the Hamiltonian as 


$$
\begin{aligned}
H= & \int \mathrm{d} \mathbf{r}\left(\frac{1}{2 \varepsilon_{0}} \Pi^{2}+\frac{1}{2 \mu_{0}}(\boldsymbol{\nabla} \times \mathbf{A})^{2}+\frac{1}{2 \rho} P^{2}+\frac{1}{2} \rho \widetilde{\omega}_{0}^{2} X^{2}\right. \\
& +\frac{1}{2 \rho} \int_{0}^{\infty} \mathrm{d} \omega Q_{\omega}^{2}+\frac{1}{2} \rho \int_{0}^{\infty} \mathrm{d} \omega \omega^{2} Y_{\omega}^{2}+\frac{\alpha}{\rho} \mathbf{A} \cdot \mathbf{P}+\frac{\alpha^{2}}{2 \rho} A^{2} \\
& \left.+\frac{1}{\rho} \int_{0}^{\infty} \mathrm{d} \omega v_{\omega} \mathbf{X} \cdot \mathbf{Q}_{\omega}\right)+\int \mathrm{d} \mathbf{r} \mathrm{d} \mathbf{r}^{\prime} \frac{\boldsymbol{\nabla} \cdot(\alpha \mathbf{X}) \boldsymbol{\nabla}^{\prime} \cdot\left(\alpha^{\prime} \mathbf{X}^{\prime}\right)}{8 \pi \varepsilon_{0}\left|\mathbf{r}-\mathbf{r}^{\prime}\right|} .
\end{aligned}
$$

We introduced the notation $\mathbf{X}^{\prime}=\mathbf{X}\left(\mathbf{r}^{\prime}\right)$, and likewise $\alpha^{\prime}$ and $\boldsymbol{\nabla}^{\prime}$. The renormalized frequency $\widetilde{\omega}_{0}(\mathbf{r})$ is defined by $\widetilde{\omega}_{0}^{2}$ $=\omega_{0}^{2}+\rho^{-2} \int_{0}^{\infty} \mathrm{d} \omega v_{\omega}^{2}$, where the integral is assumed to be finite at all positions $\mathbf{r}$.

To quantize the model we impose the usual commutation relations

$$
\begin{gathered}
{\left[\mathbf{\Pi}(\mathbf{r}), \mathbf{A}\left(\mathbf{r}^{\prime}\right)\right]=-i \hbar \boldsymbol{\delta}_{\mathrm{T}}\left(\mathbf{r}-\mathbf{r}^{\prime}\right),} \\
{\left[\mathbf{P}(\mathbf{r}), \mathbf{X}\left(\mathbf{r}^{\prime}\right)\right]=-i \hbar \mathbf{I} \delta\left(\mathbf{r}-\mathbf{r}^{\prime}\right),} \\
{\left[\mathbf{Q}_{\omega}(\mathbf{r}), \mathbf{Y}_{\omega^{\prime}}\left(\mathbf{r}^{\prime}\right)\right]=-i \hbar \delta\left(\omega-\omega^{\prime}\right) \mathbf{I} \delta\left(\mathbf{r}-\mathbf{r}^{\prime}\right),}
\end{gathered}
$$

while all other commutators of the canonical variables vanish. Here, I is the three-dimensional unit tensor, while $\boldsymbol{\delta}_{\mathrm{T}}(\mathbf{r})=\mathbf{I} \delta(\mathbf{r})+\boldsymbol{\nabla} \boldsymbol{\nabla}(4 \pi r)^{-1}$ is the transverse delta function.

With these "primary" canonical commutation relations and the relations between the potentials and field operators, one can derive the following "secondary" commutation relations

$$
\begin{gathered}
{\left[\mathbf{E}(\mathbf{r}), \mathbf{A}\left(\mathbf{r}^{\prime}\right)\right]=\frac{i \hbar}{\varepsilon_{0}} \boldsymbol{\delta}_{\mathrm{T}}\left(\mathbf{r}-\mathbf{r}^{\prime}\right),} \\
{\left[E_{j}(\mathbf{r}), B_{k}\left(\mathbf{r}^{\prime}\right)\right]=\frac{i \hbar}{\varepsilon_{0}} \epsilon_{j k l} \nabla_{l}^{\prime} \delta\left(\mathbf{r}-\mathbf{r}^{\prime}\right),}
\end{gathered}
$$

where we used Eq. (2a). Notice that these commutation relations are medium independent, because in Eq. (4) we took the electromagnetic field to be canonically independent from the material variables $\mathbf{X}$ and $\mathbf{P}$.

In the Heisenberg picture the equations of motion for the canonical variables follow by evaluating the commutators with the Hamiltonian:

$$
\begin{gathered}
\dot{\mathbf{A}}=\frac{1}{\varepsilon_{0}} \boldsymbol{\Pi}, \\
\dot{\mathbf{I}}=\frac{1}{\mu_{0}} \Delta \mathbf{A}-\left[\frac{\alpha}{\rho}(\mathbf{P}+\alpha \mathbf{A})\right]_{\mathrm{T}} \\
\dot{\mathbf{X}}=\frac{1}{\rho}(\mathbf{P}+\alpha \mathbf{A}), \\
\dot{\mathbf{P}}=-\rho \widetilde{\omega}_{0}^{2} \mathbf{X}-\frac{\alpha}{\varepsilon_{0}}[\alpha \mathbf{X}]_{\mathrm{L}}-\frac{1}{\rho} \int_{0}^{\infty} \mathrm{d} \omega v_{\omega} \mathbf{Q}_{\omega},
\end{gathered}
$$

$$
\begin{gathered}
\dot{\mathbf{Y}}_{\omega}=\frac{1}{\rho}\left(\mathbf{Q}_{\omega}+v_{\omega} \mathbf{X}\right), \\
\dot{\mathbf{Q}}_{\omega}=-\rho \omega^{2} \mathbf{Y}_{\omega} .
\end{gathered}
$$

In the second equation the subscript $\mathrm{T}$ denotes the transverse part of the vector, which is obtained by a convolution with the transverse delta function. Likewise, the subscript L in the fourth equation indicates the longitudinal part, obtained by convolving with the longitudinal delta function $\boldsymbol{\delta}_{\mathrm{L}}(\mathbf{r})=$ $-\nabla \nabla(4 \pi r)^{-1}$.

From Eqs. (6a)-(6c) one gets

$$
\Delta \mathbf{A}-c^{-2} \ddot{\mathbf{A}}=\mu_{0}\left[\frac{\alpha}{\rho}(\mathbf{P}+\alpha \mathbf{A})\right]_{\mathrm{T}}=\mu_{0}[\alpha \dot{\mathbf{X}}]_{\mathrm{T}} .
$$

This equation is equivalent to Maxwell's equation

$$
-\nabla \times \mathbf{B}+c^{-2} \dot{\mathbf{E}}=\mu_{0} \alpha \dot{\mathbf{X}},
$$

since the Poisson equation for $\Phi$ can be rewritten as

$$
[\alpha \mathbf{X}]_{\mathrm{L}}=-\varepsilon_{0} \nabla \Phi .
$$

The equations of motion [Eqs. (6c) and (6d)] for the variables of the dielectric medium yield a second-order differential equation for $\mathbf{X}$ :

$$
\rho \ddot{\mathbf{X}}+\rho \widetilde{\omega}_{0}^{2} \mathbf{X}=\alpha \dot{\mathbf{A}}-\frac{\alpha}{\varepsilon_{0}}[\alpha \mathbf{X}]_{\mathrm{L}}-\frac{1}{\rho} \int_{0}^{\infty} \mathrm{d} \omega v_{\omega} \mathbf{Q}_{\omega},
$$

or alternatively, with the use of Eq. (6e),

$$
\rho \ddot{\mathbf{X}}+\rho \omega_{0}^{2} \mathbf{X}=\alpha \dot{\mathbf{A}}-\frac{\alpha}{\varepsilon_{0}}[\alpha \mathbf{X}]_{\mathrm{L}}-\int_{0}^{\infty} \mathrm{d} \omega v_{\omega} \dot{\mathbf{Y}}_{\omega} .
$$

The change from $\widetilde{\omega}_{0}$ to $\omega_{0}$ should be noted here. The first two terms on the right-hand sides of Eqs. (10) and (11) are equal to $-\alpha \mathbf{E}$, as follows from Eq. (9).

Finally, the second-order equation of motion for the bath follows from Eq. (6e) and (6f) as

$$
\rho \ddot{\mathbf{Y}}_{\omega}+\rho \omega^{2} \mathbf{Y}_{\omega}=v_{\omega} \dot{\mathbf{X}} .
$$

The second-order equations (7), (11), and (12) determine the time evolution of the basic physical variables $\mathbf{A}, \mathbf{X}$, and $\mathbf{Y}_{\omega}$, which represent the vector potential, the dielectric polarization density and the harmonic-oscillator displacement density of the bath. If the initial conditions of $\mathbf{A}, \mathbf{X}$, and $\mathbf{Y}_{\omega}$ and their first time derivatives are given at $t=0$, these operators are known at any value of $t$, either positive or negative.

\section{LAPLACE TRANSFORMS}

Our goal for the remainder of this paper is twofold. First, we would like to determine the dynamics of the model and to find the complete time dependence of the canonical variables. Second, we want to show how the phenomenological theory emerges from our model. To that end, we must identify the elements of the phenomenological theory (dielectric function, Green function, and noise-current density) in terms of the variables from the model. Then, after their identifica- 
tion we must show that these quantities have all the desired properties that are merely postulated in the phenomenological approach.

The equations of motion of the previous section constitute a set of linear differential equations. In this section, we solve them in terms of their initial conditions, by introducing Laplace transforms. This technique has been used before in the analysis of the damped-polariton model $[15,16]$. In particular, we will derive an equation for the Laplace transform of the electric field $\mathbf{E}$, while taking care to eliminate the Laplace-transformed variables describing the dielectric medium. To that end we shall first solve the Laplacetransformed equations for the bath variables. Subsequently, these will be used to obtain an identity that relates the Laplace transforms of the polarization density and the electric field. While establishing that relationship, we shall identify the electric susceptibility in Laplace language. Finally, wave equations for the Laplace transforms of the electric field will be deduced. All equations will be valid for an arbitrary spatial dependence of the variables of the model.

For any time-dependent operator $\Omega$ the (forward) Laplace transform is defined as

$$
\bar{\Omega}(p)=\int_{0}^{\infty} \mathrm{d} t e^{-p t} \Omega(t) .
$$

Obviously, the Laplace transform contains all information on the time evolution of $\Omega$ for positive $t$. In the following we wish to determine the time evolution of the relevant operators of our model for any time, either positive or negative. Hence, we also introduce the backward Laplace transform:

$$
\breve{\Omega}(p)=\int_{0}^{\infty} \mathrm{d} t e^{-p t} \Omega(-t) .
$$

Both transforms are defined for all $p$ with $\operatorname{Re} p>0$.

Carrying out the (forward) Laplace transformation of Eqs. (6e) and (6f), and eliminating $\overline{\mathbf{Y}}_{\omega}(p)$ we find

$$
\overline{\mathbf{Q}}_{\omega}(p)=-\frac{\omega^{2}}{p^{2}+\omega^{2}} v_{\omega} \overline{\mathbf{X}}(p)+\frac{1}{p^{2}+\omega^{2}}\left[p \mathbf{Q}_{\omega}(0)-\rho \omega^{2} \mathbf{Y}_{\omega}(0)\right],
$$

with $\mathbf{Q}_{\omega}(0)$ and $\mathbf{Y}_{\omega}(0)$ the initial conditions at $t=0$. Alternatively, we could have used Eq. (12) as a starting point. Upon performing its Laplace transformation, we may eliminate the initial condition for $\dot{\mathbf{Y}}_{\omega}$ with the help of Eq. (6e). The initial condition of $\mathbf{X}$ is then found to drop out as well, so that Eq. (15) is recovered.

From Eqs. (6c) and (6d) we find analogously, after elimination of $\overline{\mathbf{P}}(p)$ :

$$
\begin{aligned}
\left(p^{2}+\widetilde{\omega}_{0}^{2}\right) \overline{\mathbf{X}}(p)= & \frac{\alpha}{\rho}\left\{p \overline{\mathbf{A}}(p)-\frac{1}{\varepsilon_{0}}[\alpha \overline{\mathbf{X}}(p)]_{\mathrm{L}}\right\} \\
& -\frac{1}{\rho^{2}} \int_{0}^{\infty} \mathrm{d} \omega v_{\omega} \overline{\mathbf{Q}}_{\omega}(p)+p \mathbf{X}(0)+\frac{1}{\rho} \mathbf{P}(0) .
\end{aligned}
$$

Insertion of Eq. (15) in the integral yields:

$$
\begin{aligned}
{\left[p^{2}\right.} & \left.+\widetilde{\omega}_{0}^{2}-\frac{1}{\rho^{2}} \int_{0}^{\infty} \mathrm{d} \omega \frac{\omega^{2} v_{\omega}^{2}}{p^{2}+\omega^{2}}\right] \overline{\mathbf{X}}(p) \\
& =\frac{\alpha}{\rho}\left\{p \overline{\mathbf{A}}(p)-\frac{1}{\varepsilon_{0}}[\alpha \overline{\mathbf{X}}(p)]_{\mathrm{L}}\right\}+p \mathbf{X}(0)+\frac{1}{\rho} \mathbf{P}(0) \\
& +\frac{1}{\rho} \int_{0}^{\infty} \mathrm{d} \omega \frac{v_{\omega}}{p^{2}+\omega^{2}}\left[\omega^{2} \mathbf{Y}_{\omega}(0)-\frac{p}{\rho} \mathbf{Q}_{\omega}(0)\right] .
\end{aligned}
$$

The first few terms at the right-hand side are related to the Laplace transform of the electric field, which reads

$$
\overline{\mathbf{E}}(p)=-p \overline{\mathbf{A}}(p)+\frac{1}{\varepsilon_{0}}[\alpha \overline{\mathbf{X}}(p)]_{\mathrm{L}}+\mathbf{A}(0) .
$$

From Eqs. (17) and (18) it follows that the Laplacetransformed polarization density $-\alpha \overline{\mathbf{X}}(p)$ is proportional to the Laplace-transformed electric field, apart from terms depending on the initial conditions. We identify the proportionality constant as the electric susceptibility in Laplace language:

$$
\bar{\chi}(p)=\frac{\alpha^{2}}{\varepsilon_{0} \rho} \frac{1}{p^{2}+\widetilde{\omega}_{0}^{2}-\rho^{-2} \int_{0}^{\infty} \mathrm{d} \omega \omega^{2} v_{\omega}^{2} /\left(p^{2}+\omega^{2}\right)} .
$$

All parameters at the right-hand side depend on position, so that the susceptibility is a space-dependent quantity. In this respect it is a generalization of the definitions in Refs. $[8,9,12,13]$, which are valid for homogeneous dielectrics. The susceptibility is an analytic function of $p$ for all $p$ with $\operatorname{Re} p>0$. Indeed, one can prove that the denominator in Eq. (19) cannot vanish for any $p$ in the right half-plane. As a consequence of its analyticity properties, the susceptibility satisfies the standard Kramers-Kronig relations which connect the real and imaginary parts of $\bar{\chi}(p)$.

After introduction of the susceptibility $\bar{\chi}(p)$ the linear relationship between $\overline{\mathbf{X}}(p)$ and $\overline{\mathbf{E}}(p)$ gets the form:

$$
\begin{aligned}
\overline{\mathbf{X}}(p)= & -\frac{\varepsilon_{0}}{\alpha} \bar{\chi}(p) \overline{\mathbf{E}}(p)+\frac{\varepsilon_{0}}{\alpha^{2}} \bar{\chi}(p)\{\alpha \mathbf{A}(0)+\rho p \mathbf{X}(0)+\mathbf{P}(0) \\
& \left.+\int_{0}^{\infty} \mathrm{d} \omega \frac{v_{\omega}}{p^{2}+\omega^{2}}\left[\omega^{2} \mathbf{Y}_{\omega}(0)-\frac{p}{\rho} \mathbf{Q}_{\omega}(0)\right]\right\} .
\end{aligned}
$$

This identity, which relates the Laplace transforms of the polarization density and the electric field, will be crucial in eliminating the dielectric variables, as we shall see presently. As expected, the relationship still depends on the initial values of all canonical variables [with the exception of $\boldsymbol{\Pi}(0)$ ].

Having succeeded in expressing the polarization density in terms of the electric field, we would like to establish a wave equation for the electric field in Laplace language. All Laplace-transformed equations, which we derived above, contain terms depending on the initial conditions. Hence, we expect that the wave equation will contain such terms as well. In fact, we shall show that owing to the presence of these terms, the wave equation will be inhomogeneous. 
To derive the wave equation in Laplace language, we have to use the remaining field-dependent equations in the set (6). However, instead of Eqs. (6a) and (6b) we prefer to employ their corollary Eq. (8), or, better still, the equation which follows by taking the time derivative of Eq. (8) and using the induction law:

$$
\boldsymbol{\nabla} \times(\boldsymbol{\nabla} \times \mathbf{E})+c^{-2} \ddot{\mathbf{E}}=\mu_{0} \alpha \ddot{\mathbf{X}} .
$$

After a (forward) Laplace transformation we get

$$
\begin{aligned}
& \boldsymbol{\nabla} \times {[\boldsymbol{\nabla} \times \overline{\mathbf{E}}(p)]+c^{-2} p^{2} \overline{\mathbf{E}}(p)-\mu_{0} \alpha p^{2} \overline{\mathbf{X}}(p) } \\
&=c^{-2} \dot{\mathbf{E}}(0)+c^{-2} p \mathbf{E}(0)-\mu_{0} \alpha \dot{\mathbf{X}}(0)-\mu_{0} \alpha p \mathbf{X}(0) .
\end{aligned}
$$

As before, the introduction of Laplace transforms has led to terms depending on the initial conditions. We wish to express these in terms of the canonical variables at $t=0$. At the righthand side we use Eq. (6c) for $t=0$ to rewrite $\dot{\mathbf{X}}(0)$ :

$$
\dot{\mathbf{X}}(0)=\frac{\alpha}{\rho} \mathbf{A}(0)+\frac{1}{\rho} \mathbf{P}(0) .
$$

Upon inserting this relation in Eq. (8) for $t=0$ we find

$$
\dot{\mathbf{E}}(0)=c^{2} \boldsymbol{\nabla} \times[\boldsymbol{\nabla} \times \mathbf{A}(0)]+\frac{\alpha^{2}}{\varepsilon_{0} \rho} \mathbf{A}(0)+\frac{\alpha}{\varepsilon_{0} \rho} \mathbf{P}(0) .
$$

Furthermore, we use Eq. (6a) and (9) to write the electric field at $t=0$ as

$$
\mathbf{E}(0)=-\frac{1}{\varepsilon_{0}} \Pi(0)+\frac{1}{\varepsilon_{0}}[\alpha \mathbf{X}(0)]_{\mathrm{L}} .
$$

Substituting Eqs. (23)-(25) on the right-hand side of Eq. (22), and using the expression (20) for $\overline{\mathbf{X}}(p)$ in the last term at the left-hand side, we finally arrive at the Laplacetransformed wave equation for the electric field in the form:

$$
\boldsymbol{\nabla} \times[\boldsymbol{\nabla} \times \overline{\mathbf{E}}(p)]+c^{-2} p^{2} \bar{\varepsilon}(p) \overline{\mathbf{E}}(p)=-\mu_{0} p \overline{\mathbf{J}}(p),
$$

with $\bar{\varepsilon}(p)=1+\bar{\chi}(p)$ the (relative) electric permeability in Laplace language.

The differential equation (26) in Laplace language is an inhomogeneous wave equation. The source term at the righthand side depends on the initial conditions at time $t=0$ :

$$
\begin{aligned}
\overline{\mathbf{J}}(p)= & -\frac{1}{\mu_{0} p} \boldsymbol{\nabla} \times[\boldsymbol{\nabla} \times \mathbf{A}(0)]-\varepsilon_{0} p \bar{\chi}(p) \mathbf{A}(0)+\boldsymbol{\Pi}(0) \\
& +\alpha\left[1-\frac{\varepsilon_{0} \rho}{\alpha^{2}} p^{2} \bar{\chi}(p)\right] \mathbf{X}(0)-[\alpha \mathbf{X}(0)]_{\mathrm{L}}-\frac{\varepsilon_{0}}{\alpha} p \bar{\chi}(p) \mathbf{P}(0) \\
& -\frac{\varepsilon_{0}}{\alpha} p \bar{\chi}(p) \int_{0}^{\infty} \mathrm{d} \omega \frac{v_{\omega}}{p^{2}+\omega^{2}}\left[\omega^{2} \mathbf{Y}_{\omega}(0)-\frac{p}{\rho} \mathbf{Q}_{\omega}(0)\right] .
\end{aligned}
$$

In principle, the electric field in the inhomogeneous wave equation (26) can be solved in terms of its source. Upon performing the inverse Laplace transformation, one then gets an expression for the electric field at any later time after $t$ $=0$. However, as noted above, we also need the electric field (and the other variables of the model) for all times prior to $t=0$. To find that information we have to derive the corresponding wave equation for the backward Laplace transform of the electric field. We shall proceed along the same lines as above.

The backward Laplace transforms of Eqs. (6e) and (6f) yield on a par with Eq. (15):

$$
\breve{\mathbf{Q}}_{\omega}(p)=-\frac{\omega^{2}}{p^{2}+\omega^{2}} v_{\omega} \breve{\mathbf{X}}(p)+\frac{1}{p^{2}+\omega^{2}}\left[p \mathbf{Q}_{\omega}(0)+\rho \omega^{2} \mathbf{Y}_{\omega}(0)\right]
$$

where the change of sign in the last term should be noted. Using this equation and following the same steps as above, we find the counterpart of Eq. (20) as

$$
\begin{aligned}
\breve{\mathbf{X}}(p)= & -\frac{\varepsilon_{0}}{\alpha} \bar{\chi}(p) \breve{\mathbf{E}}(p)+\frac{\varepsilon_{0}}{\alpha^{2}} \bar{\chi}(p)\{-\alpha \mathbf{A}(0)+\rho p \mathbf{X}(0)-\mathbf{P}(0) \\
& \left.-\int_{0}^{\infty} \mathrm{d} \omega \frac{v_{\omega}}{p^{2}+\omega^{2}}\left[\omega^{2} \mathbf{Y}_{\omega}(0)+\frac{p}{\rho} \mathbf{Q}_{\omega}(0)\right]\right\} .
\end{aligned}
$$

The susceptibility is given by Eq. (19), as before. The backward Laplace transform of the electric field is slightly different from Eq. (18):

$$
\breve{\mathbf{E}}(p)=p \breve{\mathbf{A}}(p)+\frac{1}{\varepsilon_{0}}[\alpha \breve{\mathbf{X}}(p)]_{\mathrm{L}}-\mathbf{A}(0) .
$$

Here, as in Eq. (29), several terms have changed sign. Finally, turning to the field equation, we obtain from Eq. (21) after a backward Laplace transformation:

$$
\begin{aligned}
\boldsymbol{\nabla} \times & {[\boldsymbol{\nabla} \times \breve{\mathbf{E}}(p)]+c^{-2} p^{2} \breve{\mathbf{E}}(p)-\mu_{0} \alpha p^{2} \breve{\mathbf{X}}(p) } \\
& =-c^{-2} \dot{\mathbf{E}}(0)+c^{-2} p \mathbf{E}(0)+\mu_{0} \alpha \dot{\mathbf{X}}(0)-\mu_{0} \alpha p \mathbf{X}(0) .
\end{aligned}
$$

Substitution of Eqs. (23)-(25) yields as the analog of Eq. (26):

$$
\boldsymbol{\nabla} \times[\boldsymbol{\nabla} \times \breve{\mathbf{E}}(p)]+c^{-2} p^{2} \bar{\varepsilon}(p) \breve{\mathbf{E}}(p)=\mu_{0} p \breve{\mathbf{J}}(p) .
$$

The source term in this inhomogeneous wave equation is:

$$
\begin{aligned}
\breve{\mathbf{J}}(p)= & -\frac{1}{\mu_{0} p} \boldsymbol{\nabla} \times[\boldsymbol{\nabla} \times \mathbf{A}(0)]-\varepsilon_{0} p \bar{\chi}(p) \mathbf{A}(0)-\boldsymbol{\Pi}(0) \\
& -\alpha\left[1-\frac{\varepsilon_{0} \rho}{\alpha^{2}} p^{2} \bar{\chi}(p)\right] \mathbf{X}(0)+[\alpha \mathbf{X}(0)]_{\mathrm{L}}-\frac{\varepsilon_{0}}{\alpha} p \bar{\chi}(p) \mathbf{P}(0) \\
& -\frac{\varepsilon_{0}}{\alpha} p \bar{\chi}(p) \int_{0}^{\infty} \mathrm{d} \omega \frac{v_{\omega}}{p^{2}+\omega^{2}}\left[\omega^{2} \mathbf{Y}_{\omega}(0)+\frac{p}{\rho} \mathbf{Q}_{\omega}(0)\right]
\end{aligned}
$$

As expected, several terms have changed sign as compared to Eq. (27). For future convenience we have chosen the sign on the right-hand side of Eq. (32) to be the opposite of that in Eq. (26). 
The main results in this section are the expressions (27) and (33) for $\overline{\mathbf{J}}(p)$ and $\breve{\mathbf{J}}(p)$. These are the source terms of the Laplace-transformed wave equations (26) and (32). It should be noted that these source terms are not defined as the forward and backward Laplace transforms of some operator $\mathbf{J}(\mathbf{r}, t)$, although their notation might suggest otherwise. As a consequence, their properties differ from other pairs of operators, like $\overline{\mathbf{E}}(p)$ and $\breve{\mathbf{E}}(p)$. In Sec. VI we shall return to this point.

\section{GREEN FUNCTIONS AND SOLUTIONS OF WAVE EQUATIONS}

In Sec. III we have seen that both the forward and the backward Laplace transform of the electric field satisfy a wave equation with a source term. To solve these equations we introduce tensorial Green functions in Laplace language. The Green function associated to the wave equations (26) and (32) is defined as the solution of the differential equation $[22,23,26]$

$$
-\nabla \times\left[\nabla \times \overline{\mathbf{G}}\left(\mathbf{r}, \mathbf{r}^{\prime}, p\right)\right]-\frac{p^{2}}{c^{2}} \bar{\varepsilon}(\mathbf{r}, p) \overline{\mathbf{G}}\left(\mathbf{r}, \mathbf{r}^{\prime}, p\right)=\mathbf{I} \delta\left(\mathbf{r}-\mathbf{r}^{\prime}\right),
$$

where for clarity we reintroduced the spatial argument of the permeability. The function $\mathbf{G}\left(\mathbf{r}, \mathbf{r}^{\prime}, p\right)$ is the forward Laplace transform of the standard retarded Green function of macroscopic electrodynamics. It is also equal to the backward Laplace transform of the advanced Green function. The Green function is analytic for all $p$ in the half-plane with $\operatorname{Re} p>0$, as is the susceptibility $\bar{\chi}(\mathbf{r}, p)$ [23]. The defining equation (34) can be read as the statement that the Green function is the inverse of the operator $-[\nabla \times(\nabla \times)]$ $-c^{-2} p^{2} \bar{\varepsilon}(\mathbf{r}, p)(\mathbf{I} \cdot)$, which is a symmetric differential operator in the space of square-integrable vector functions. Owing to the symmetry the Green function satisfies the reciprocity relation

$$
\left[\overline{\mathbf{G}}\left(\mathbf{r}, \mathbf{r}^{\prime}, p\right)\right]_{i j}=\left[\overline{\mathbf{G}}\left(\mathbf{r}^{\prime}, \mathbf{r}, p\right)\right]_{j i} .
$$

The adjoint equation of (34) reads

$$
-\left[\overline{\mathbf{G}}\left(\mathbf{r}, \mathbf{r}^{\prime}, p\right) \times \overleftarrow{\nabla}^{\prime}\right] \times \overleftarrow{\nabla}^{\prime}-\frac{p^{2}}{c^{2}} \bar{\varepsilon}\left(\mathbf{r}^{\prime}, p\right) \overline{\mathbf{G}}\left(\mathbf{r}, \mathbf{r}^{\prime}, p\right)=\mathbf{I} \delta\left(\mathbf{r}-\mathbf{r}^{\prime}\right),
$$

where the spatial derivatives operate to the left.

In terms of the Green function, the solution of Eq. (26) for the Laplace transform of the electric field is

$$
\overline{\mathbf{E}}(\mathbf{r}, p)=\mu_{0} p \int d \mathbf{r}^{\prime} \overline{\mathbf{G}}\left(\mathbf{r}, \mathbf{r}^{\prime}, p\right) \cdot \overline{\mathbf{J}}\left(\mathbf{r}^{\prime}, p\right)
$$

Likewise, the backward Laplace transform of the field follows from Eq. (32) as

$$
\breve{\mathbf{E}}(\mathbf{r}, p)=-\mu_{0} p \int d \mathbf{r}^{\prime} \overline{\mathbf{G}}\left(\mathbf{r}, \mathbf{r}^{\prime}, p\right) \cdot \breve{\mathbf{J}}\left(\mathbf{r}^{\prime}, p\right) .
$$

The two integral representations [Eqs. (37) and (38)] for the forward and the backward Laplace transform of the electric field contain all information that is needed to express the electric field at time $t$ in terms of the initial conditions of the canonical variables. The latter show up explicitly when the expressions (27) and (33) are substituted in the integrals.

The time-dependent electric field is obtained from the integral representations by an inverse Laplace transformation. From Eq. (37) we get the electric field for $t>0$ :

$$
\begin{aligned}
\mathbf{E}(\mathbf{r}, t)= & -\frac{i \mu_{0}}{2 \pi} \int_{-\infty}^{\infty} d \omega e^{-i \omega t} \omega \\
& \times \int d \mathbf{r}^{\prime} \overline{\mathbf{G}}\left(\mathbf{r}, \mathbf{r}^{\prime},-i \omega+0\right) \cdot \overline{\mathbf{J}}\left(\mathbf{r}^{\prime},-i \omega+0\right),
\end{aligned}
$$

where we changed the integration variable from $p$ on the right half-plane to $-i \omega+\eta$, with a small but positive $\eta$. We formally replace $\eta$ by 0 , so that $-i \omega+\eta$ becomes $-i \omega+0$.

The electric field for $t<0$ is obtained from the inverse Laplace transform of Eq. (38):

$$
\begin{aligned}
\mathbf{E}(\mathbf{r}, t)= & -\frac{i \mu_{0}}{2 \pi} \int_{-\infty}^{\infty} d \omega e^{-i \omega t} \omega \\
& \times \int d \mathbf{r}^{\prime} \overline{\mathbf{G}}\left(\mathbf{r}, \mathbf{r}^{\prime}, i \omega+0\right) \cdot \breve{\mathbf{J}}\left(\mathbf{r}^{\prime}, i \omega+0\right) .
\end{aligned}
$$

The $\omega$-dependent integrand in Eq. (39) is analytic for all $\omega$ in the upper half-plane. Hence, the integral over $\omega$ vanishes for negative $t$, since the $\omega$-contour can be closed in the upper half-plane for $t<0$. Likewise, the right-hand side of Eq. (40) is zero for positive $t$. As a consequence, one may combine the two expressions into a single one, which is valid for all $t$ :

$$
\mathbf{E}(\mathbf{r}, t)=\int_{0}^{\infty} d \omega e^{-i \omega t} \mathbf{E}^{(+)}(\mathbf{r}, \omega)+\text { H.c. }
$$

with the positive-frequency Fourier component:

$$
\begin{aligned}
\mathbf{E}^{(+)}(\mathbf{r}, \omega)= & -\frac{i \mu_{0} \omega}{2 \pi} \int d \mathbf{r}^{\prime}\left[\overline{\mathbf{G}}\left(\mathbf{r}, \mathbf{r}^{\prime},-i \omega+0\right) \cdot \overline{\mathbf{J}}\left(\mathbf{r}^{\prime},-i \omega+0\right)\right. \\
& \left.+\overline{\mathbf{G}}\left(\mathbf{r}, \mathbf{r}^{\prime}, i \omega+0\right) \cdot \breve{\mathbf{J}}\left(\mathbf{r}^{\prime}, i \omega+0\right)\right] .
\end{aligned}
$$

Alternatively, one may write

$$
\mathbf{E}^{(+)}(\mathbf{r}, \omega)=\frac{1}{2 \pi}[\overline{\mathbf{E}}(\mathbf{r},-i \omega+0)+\breve{\mathbf{E}}(\mathbf{r}, i \omega+0)],
$$

as follows by going back to Eqs. (37) and (38).

We have succeeded now in obtaining the full time dependence of the electric-field operator. As the representation Eq. (41) shows, the field is a linear superposition of contributions, each with its own time dependence, and with a weight that is determined by the positive-frequency Fourier component Eq. (42). The latter is itself a linear combination 
of the canonical variables at $t=0$, as follows from Eqs. (27) and (33).

The representation (41) is valid for all $t$. Hence, consistency demands that the right-hand side of Eq. (41) should reduce to the electric field (25) in the limit $t \rightarrow 0$. In Appendix A we show that this is indeed the case. It turns out that the proof depends on the validity of several sum rules for the Green function. The latter hold true as a consequence of the analyticity of the Green function and of its asymptotic behavior for large frequencies.

Having determined the positive-frequency Fourier component of the electric-field operator, we may try and find the differential equation which it satisfies. Of course, one expects this equation to be of similar form as in Eq. (26) and (32), with a frequency $\omega$ instead of the Laplace variable $p$. Therefore, we introduce the permeability in the frequency domain as $\varepsilon(\mathbf{r}, \omega)=\bar{\varepsilon}(\mathbf{r},-i \omega+0)$, for real $\omega$. Likewise, we will write $\bar{\chi}(\mathbf{r},-i \omega+0)$ as $\chi(\mathbf{r}, \omega)$. In terms of the permeability $\varepsilon(\mathbf{r}, \omega)$ we may define the differential operator $-[\boldsymbol{\nabla}$ $\times(\boldsymbol{\nabla} \times)]+c^{-2} \omega^{2} \varepsilon(\mathbf{r}, \omega)(\mathbf{I} \cdot)$. The positive-frequency Fourier component of the electric field satisfies a differential equation containing this operator:

$$
\begin{aligned}
-\boldsymbol{\nabla} & \times\left[\boldsymbol{\nabla} \times \mathbf{E}^{(+)}(\mathbf{r}, \omega)\right]+\frac{\omega^{2}}{c^{2}} \varepsilon(\mathbf{r}, \omega) \mathbf{E}^{(+)}(\mathbf{r}, \omega) \\
& =-i \mu_{0} \omega \mathbf{J}(\mathbf{r}, \omega) .
\end{aligned}
$$

As expected, the differential equation is inhomogeneous, like Eq. (26) and (32). Apart from a trivial factor, the right-hand side contains a source term $\mathbf{J}(\mathbf{r}, \omega)$. Since the positivefrequency part $\mathbf{E}^{(+)}(\mathbf{r}, \omega)$ of the electric field is known from Eq. (42), (27), and (33), the differential equation (44) may serve as the definition of the operator $\mathbf{J}(\mathbf{r}, \omega)$. Defining $\mathbf{J}(\mathbf{r}, \omega)$ in this way we try to make contact with the phenomenological quantization procedure, in which an equation of the same form as Eq. (44) plays an important role [17-23]. Although the equations have the same form in the two theories, their interpretation is rather different. In the phenomenological quantization procedure neither $\mathbf{E}^{(+)}(\mathbf{r}, \omega)$ nor $\mathbf{J}(\mathbf{r}, \omega)$ are known at first. To obtain a well-defined theory one has to postulate several properties of $\mathbf{J}(\mathbf{r}, \omega)$. In particular, one postulates an identity for the commutator of $\mathbf{J}(\mathbf{r}, \omega)$ with its Hermitian conjugate. Only after doing so does one arrive at a meaningful theory. In the present theory we are able to derive the algebraic properties of $\mathbf{J}(\mathbf{r}, \omega)$, as we shall see later on.

As in the phenomenological theory, the operator $\mathbf{J}(\mathbf{r}, \omega)$ may be interpreted as a (frequency-dependent) noise-current density. Its form still needs to be elaborated in more detail. By substituting Eq. (42) at the left-hand side of Eq. (44) and using the definition (34) of the Green function to eliminate the differential operators, we get

$$
\begin{aligned}
\mathbf{J}(\mathbf{r}, \omega)= & \frac{1}{2 \pi}[\overline{\mathbf{J}}(\mathbf{r},-i \omega+0)+\breve{\mathbf{J}}(\mathbf{r}, i \omega+0)] \\
& +\frac{\omega^{2}}{2 \pi c^{2}}[\bar{\chi}(\mathbf{r},-i \omega+0)-\bar{\chi}(\mathbf{r}, i \omega+0)] \\
& \times \int d \mathbf{r}^{\prime} \overline{\mathbf{G}}\left(\mathbf{r}, \mathbf{r}^{\prime}, i \omega+0\right) \cdot \breve{\mathbf{J}}\left(\mathbf{r}^{\prime}, i \omega+0\right) .
\end{aligned}
$$

It turns out that the operator $\mathbf{J}(\mathbf{r}, \omega)$ has a more complicated structure than the positive-frequency Fourier component (43) of the electric field. Whereas the first two terms on the righthand side have the expected form, an additional contribution shows up, which has arisen from the second term of Eq. (42). In fact, the operator acting on the electric field in Eq. (44) is the inverse of $\overline{\mathbf{G}}\left(\mathbf{r}, \mathbf{r}^{\prime},-i \omega+0\right)$, but not of $\overline{\mathbf{G}}\left(\mathbf{r}, \mathbf{r}^{\prime}, i \omega+0\right)$ : the frequency arguments of the permeability do not match in the latter case. The additional term is proportional to the imaginary part of the susceptibility $\bar{\chi}(\mathbf{r},-i \omega+0)$.

The operator $\mathbf{J}(\mathbf{r}, \omega)$ will play an important role in the following. Before evaluating it explicitly in terms of the canonical variables, we will show that it can also be obtained in a different way. Let us consider, on a par with Eq. (43), the positive-frequency Fourier component of $\mathbf{X}$. As one might suppose that it is proportional to $\mathbf{E}^{(+)}$, with a proportionality constant determined by the susceptibility, we will focus on the combination

$$
\begin{aligned}
-\alpha & \mathbf{X}^{(+)}(\mathbf{r}, \omega)-\varepsilon_{0} \chi(\mathbf{r}, \omega) \mathbf{E}^{(+)}(\mathbf{r}, \omega) \\
& =-\frac{\alpha}{2 \pi}[\overline{\mathbf{X}}(\mathbf{r},-i \omega+0)+\breve{\mathbf{X}}(\mathbf{r}, i \omega+0)] \\
& -\frac{\varepsilon_{0}}{2 \pi} \bar{\chi}(\mathbf{r},-i \omega+0)[\overline{\mathbf{E}}(\mathbf{r},-i \omega+0)+\breve{\mathbf{E}}(\mathbf{r}, i \omega+0)] .
\end{aligned}
$$

Comparing Eqs. (22) and (26) and taking $p=-i \omega+0$ we infer that the forward Laplace transforms satisfy the identity:

$$
\begin{aligned}
& -\alpha \overline{\mathbf{X}}(-i \omega+0)-\varepsilon_{0} \bar{\chi}(-i \omega+0) \overline{\mathbf{E}}(-i \omega+0) \\
& \quad=\frac{i}{\omega} \overline{\mathbf{J}}(-i \omega+0)-\frac{1}{\omega^{2}}\left[-\alpha \dot{\mathbf{X}}(0)+\varepsilon_{0} \dot{\mathbf{E}}(0)\right] \\
& \quad+\frac{i}{\omega}\left[-\alpha \mathbf{X}(0)+\varepsilon_{0} \mathbf{E}(0)\right],
\end{aligned}
$$

where we suppressed the dependence on $\mathbf{r}$ for the moment. Likewise, from Eqs. (31) and (32) we derive for the backward Laplace transforms:

$$
\begin{aligned}
-\alpha \breve{\mathbf{X}}(i \omega+0)-\varepsilon_{0} \bar{\chi}(i \omega+0) \breve{\mathbf{E}}(i \omega+0) \\
\quad=\frac{i}{\omega} \breve{\mathbf{J}}(i \omega+0)+\frac{1}{\omega^{2}}\left[-\alpha \dot{\mathbf{X}}(0)+\varepsilon_{0} \dot{\mathbf{E}}(0)\right] \\
-\frac{i}{\omega}\left[-\alpha \mathbf{X}(0)+\varepsilon_{0} \mathbf{E}(0)\right],
\end{aligned}
$$

where we note that the frequency argument in the susceptibility in front of $\breve{\mathbf{E}}$ differs from that in the corresponding term in Eq. (47). Adding the right-hand sides of Eqs. (47) and (48) we see that all terms depending on the operators at $t=0$ drop out. The resulting equality can be used to evaluate the right-hand side of Eq. (46), if the susceptibility in front of $\breve{\mathbf{E}}$ in Eq. (48) is changed to $\bar{\chi}(-i \omega+0)$ by hand. The correction term that is brought about in this way, can be rewritten by means of Eq. (38). Finally, we arrive at the identity: 


$$
-\alpha \mathbf{X}^{(+)}(\mathbf{r}, \omega)-\varepsilon_{0} \chi(\mathbf{r}, \omega) \mathbf{E}^{(+)}(\mathbf{r}, \omega)=\frac{i}{\omega} \mathbf{J}(\mathbf{r}, \omega) .
$$

Hence, the noise-current density $\mathbf{J}(\mathbf{r}, \omega)$ can also be found as the difference of the positive-frequency Fourier component of the polarization density $-\alpha(\mathbf{r}) \mathbf{X}(\mathbf{r}, t)$ and $\varepsilon_{0} \chi(\mathbf{r}, \omega)$ times the positive-frequency Fourier component of the electric field $\mathbf{E}(\mathbf{r}, t)$, apart from a trivial factor $i / \omega$. Hence, the noisecurrent density is due to a noise term in the polarization density, as has been noted for the homogeneous dampedpolariton model [9], and in the context of the phenomenological quantization scheme $[22,26,36]$. In the present model the noise-current density is a specific linear combination of the canonical variables, as we shall see below.

\section{EVALUATION OF THE NOISE-CURRENT DENSITY}

The expression (45) for the noise-current density $\mathbf{J}(\mathbf{r}, \omega)$ is rather formal. It depends on the forward and the backward Laplace transforms $\overline{\mathbf{J}}$ and $\breve{\mathbf{J}}$. In Sec. III these have been given in terms of the canonical variables. By using Eqs. (27) and (33), we are able to express $\mathbf{J}(\mathbf{r}, \omega)$ in the canonical variables as well.

We start by evaluating the first two contributions at the right-hand side of Eq. (45). After substitution of Eqs. (27) and (33) and adding the two contributions, several terms are found to drop out. The remaining terms are closely related. As before, we shall write $\bar{\chi}(\mathbf{r},-i \omega+0)$ as $\chi(\mathbf{r}, \omega)$, and, correspondingly, $\bar{\chi}(\mathbf{r}, i \omega+0)$ as its complex conjugate $\chi^{*}(\mathbf{r}, \omega)$. Furthermore, the imaginary part of $\chi(\mathbf{r}, \omega)$ will be written as $\chi_{i}(\mathbf{r}, \omega)$. Using these notations, we find from the first two terms of Eq. (45):

$$
\begin{aligned}
\frac{1}{2 \pi}[ & \overline{\mathbf{J}}(\mathbf{r},-i \omega+0)+\breve{\mathbf{J}}(\mathbf{r}, i \omega+0)] \\
& =-\frac{\varepsilon_{0}}{\pi} \omega \chi_{i}(\mathbf{r}, \omega) \mathbf{A}(\mathbf{r}, 0)+\frac{i \varepsilon_{0} \rho}{\pi \alpha} \omega^{2} \chi_{i}(\mathbf{r}, \omega) \mathbf{X}(\mathbf{r}, 0) \\
& -\frac{\varepsilon_{0}}{\pi \alpha} \omega \chi_{i}(\mathbf{r}, \omega) \mathbf{P}(\mathbf{r}, 0)+\frac{i \varepsilon_{0}}{2 \pi \alpha} \omega \chi(\mathbf{r}, \omega) \\
& \times \int_{0}^{\infty} d \omega^{\prime} \frac{v_{\omega^{\prime}}}{\omega^{\prime 2}-(\omega+i 0)^{2}}\left[\omega^{\prime 2} \mathbf{Y}_{\omega^{\prime}}(\mathbf{r}, 0)+\frac{i \omega}{\rho} \mathbf{Q}_{\omega^{\prime}}(\mathbf{r}, 0)\right] \\
& -\frac{i \varepsilon_{0}}{2 \pi \alpha} \omega \chi^{*}(\mathbf{r}, \omega) \int_{0}^{\infty} d \omega^{\prime} \frac{v_{\omega^{\prime}}}{\omega^{\prime 2}-(\omega-i 0)^{2}} \\
& \times\left[\omega^{\prime 2} \mathbf{Y}_{\omega^{\prime}}(\mathbf{r}, 0)+\frac{i \omega}{\rho} \mathbf{Q}_{\omega^{\prime}}(\mathbf{r}, 0)\right],
\end{aligned}
$$

where all canonical variables are taken at $t=0$. It should be noted that the two integral terms are not the Hermitian conjugates of each other, since the signs of the terms with $\mathbf{Q}_{\omega^{\prime}}$ do not match.

In the integral term of Eq. (45) we have to insert Eq. (33) for $p=i \omega+0$. The term with the spatial derivatives of the vector potential does not drop out now, as it did in Eq. (50). It can be evaluated by a partial integration in $\mathbf{r}^{\prime}$, which leads to an expression with derivatives acting on the Green function. Evaluating these by using Eq. (36), we arrive at the following two terms:

$$
\begin{aligned}
& -\frac{\varepsilon_{0}}{\pi c^{2}} \omega^{3} \chi_{i}(\mathbf{r}, \omega) \int d \mathbf{r}^{\prime} \varepsilon^{*}\left(\mathbf{r}^{\prime}, \omega\right) \mathbf{G}^{*}\left(\mathbf{r}, \mathbf{r}^{\prime}, \omega\right) \cdot \mathbf{A}\left(\mathbf{r}^{\prime}, 0\right) \\
& \quad+\frac{\varepsilon_{0}}{\pi} \omega \chi_{i}(\mathbf{r}, \omega) \mathbf{A}(\mathbf{r}, 0)
\end{aligned}
$$

Here we introduced the notation $\mathbf{G}\left(\mathbf{r}, \mathbf{r}^{\prime}, \omega\right)=\overline{\mathbf{G}}\left(\mathbf{r}, \mathbf{r}^{\prime},-i \omega\right.$ $+0)$ [and hence $\mathbf{G}^{*}\left(\mathbf{r}, \mathbf{r}^{\prime}, \omega\right)=\overline{\mathbf{G}}\left(\mathbf{r}, \mathbf{r}^{\prime}, i \omega+0\right)$ as well], in analogy to the notations for $\varepsilon$ and $\chi$. The final term in Eq. (51) cancels the first term in Eq. (50). Part of the integral term in Eq. (51) (namely, with $\chi^{*}$ instead of $\varepsilon^{*}$ ) drops out as well, when the contribution from the second term in Eq. (33) is taken into account.

Collecting all terms, we arrive at the following result for the noise-current density:

$$
\begin{aligned}
\mathbf{J}(\mathbf{r}, \omega)= & \int d \mathbf{r}^{\prime}\left\{\mathbf{C}_{A}\left(\mathbf{r}, \mathbf{r}^{\prime}, \omega\right) \cdot \mathbf{A}\left(\mathbf{r}^{\prime}, 0\right)\right. \\
& +\mathbf{C}_{\Pi}\left(\mathbf{r}, \mathbf{r}^{\prime}, \omega\right) \cdot \mathbf{\Pi}\left(\mathbf{r}^{\prime}, 0\right)+\mathbf{C}_{X}\left(\mathbf{r}, \mathbf{r}^{\prime}, \omega\right) \cdot \mathbf{X}\left(\mathbf{r}^{\prime}, 0\right) \\
& +\mathbf{C}_{P}\left(\mathbf{r}, \mathbf{r}^{\prime}, \omega\right) \cdot \mathbf{P}\left(\mathbf{r}^{\prime}, 0\right) \\
& +\int_{0}^{\infty} d \omega^{\prime} \mathbf{C}_{Y Q}\left(\mathbf{r}, \mathbf{r}^{\prime}, \omega, \omega^{\prime}\right) \cdot\left[\omega^{\prime 2} \mathbf{Y}_{\omega^{\prime}}\left(\mathbf{r}^{\prime}, 0\right)\right. \\
& \left.\left.+\frac{i \omega}{\rho^{\prime}} \mathbf{Q}_{\omega^{\prime}}\left(\mathbf{r}^{\prime}, 0\right)\right]\right\}
\end{aligned}
$$

The (tensorial) coefficients have the following form:

$$
\begin{gathered}
\mathbf{c}_{A}\left(\mathbf{r}, \mathbf{r}^{\prime}, \omega\right)=-\frac{\varepsilon_{0}}{\pi c^{2}} \omega^{3} \chi_{i}(\mathbf{r}, \omega) \mathbf{G}_{\mathrm{T}^{\prime}}^{*}\left(\mathbf{r}, \mathbf{r}^{\prime}, \omega\right), \\
\mathbf{C}_{\Pi}\left(\mathbf{r}, \mathbf{r}^{\prime}, \omega\right)=-\frac{i}{\pi c^{2}} \omega^{2} \chi_{i}(\mathbf{r}, \omega) \mathbf{G}_{\mathrm{T}^{\prime}}^{*}\left(\mathbf{r}, \mathbf{r}^{\prime}, \omega\right), \\
\mathbf{C}_{X}\left(\mathbf{r}, \mathbf{r}^{\prime}, \omega\right)= \\
-\frac{i \varepsilon_{0} \rho}{\pi \alpha} \omega^{2} \chi_{i}(\mathbf{r}, \omega) \mathbf{I} \delta\left(\mathbf{r}-\mathbf{r}^{\prime}\right) \\
-\frac{i \alpha^{\prime}}{\pi c^{2}} \omega^{2} \chi_{i}(\mathbf{r}, \omega) \mathbf{G}_{\mathrm{T}^{\prime}}^{*}\left(\mathbf{r}, \mathbf{r}^{\prime}, \omega\right) \\
-\frac{i \varepsilon_{0} \rho^{\prime}}{\pi c^{2} \alpha^{\prime}} \omega^{4} \chi_{i}(\mathbf{r}, \omega) \chi^{*}\left(\mathbf{r}^{\prime}, \omega\right) \mathbf{G}^{*}\left(\mathbf{r}, \mathbf{r}^{\prime}, \omega\right),
\end{gathered}
$$

$$
\begin{aligned}
\mathbf{c}_{P}\left(\mathbf{r}, \mathbf{r}^{\prime}, \omega\right)= & -\frac{\varepsilon_{0}}{\pi \alpha} \omega \chi_{i}(\mathbf{r}, \omega) \mathbf{I} \delta\left(\mathbf{r}-\mathbf{r}^{\prime}\right) \\
& +\frac{\varepsilon_{0}}{\pi c^{2} \alpha^{\prime}} \omega^{3} \chi_{i}(\mathbf{r}, \omega) \chi^{*}\left(\mathbf{r}^{\prime}, \omega\right) \mathbf{G}^{*}\left(\mathbf{r}, \mathbf{r}^{\prime}, \omega\right),
\end{aligned}
$$




$$
\begin{aligned}
\mathbf{c}_{Y Q}\left(\mathbf{r}, \mathbf{r}^{\prime}, \omega, \omega^{\prime}\right)= & -\frac{\varepsilon_{0}}{\pi \alpha} \operatorname{Im}\left[\frac{\omega v_{\omega^{\prime}}}{\omega^{\prime 2}-(\omega+i 0)^{2}} \chi(\mathbf{r}, \omega)\right] \\
& \times \mathbf{I} \delta\left(\mathbf{r}-\mathbf{r}^{\prime}\right)+\frac{\varepsilon_{0}}{\pi c^{2} \alpha^{\prime}} \frac{\omega^{3} v_{\omega^{\prime}}^{\prime}}{\omega^{\prime 2}-(\omega-i 0)^{2}} \\
& \times \chi_{i}(\mathbf{r}, \omega) \chi^{*}\left(\mathbf{r}^{\prime}, \omega\right) \mathbf{G}^{*}\left(\mathbf{r}, \mathbf{r}^{\prime}, \omega\right) .
\end{aligned}
$$

In the first three formulas the complex conjugate of the Green function $\mathbf{G}_{\mathrm{T}^{\prime}}\left(\mathbf{r}, \mathbf{r}^{\prime}, \omega\right)$ appears. It is the transverse part of $\mathbf{G}\left(\mathbf{r}, \mathbf{r}^{\prime}, \omega\right)$ with respect to $\mathbf{r}^{\prime}$, which is defined by the convolution $\int d \mathbf{r}^{\prime \prime} \mathbf{G}\left(\mathbf{r}, \mathbf{r}^{\prime \prime}, \omega\right) \cdot \delta_{\mathrm{T}}\left(\mathbf{r}^{\prime \prime}-\mathbf{r}^{\prime}\right)$. In the last term of Eq. (53e) the symbol $v_{\omega^{\prime}}^{\prime}$ denotes the bath coupling parameter at the position $\mathbf{r}^{\prime}$ (and the frequency $\omega^{\prime}$ ). Furthermore, $\alpha^{\prime}$ and $\rho^{\prime}$ stand for $\alpha\left(\mathbf{r}^{\prime}\right)$ and $\rho\left(\mathbf{r}^{\prime}\right)$, respectively.

The coefficients in Eq. (53) can be interpreted as commutators. In fact, from Eq. (4) we infer

$$
\begin{gathered}
\frac{i}{\hbar}\left[\mathbf{J}(\mathbf{r}, \omega), \Pi\left(\mathbf{r}^{\prime}, 0\right)\right]=-\mathbf{c}_{A}\left(\mathbf{r}, \mathbf{r}^{\prime}, \omega\right), \\
\frac{i}{\hbar}\left[\mathbf{J}(\mathbf{r}, \omega), \mathbf{A}\left(\mathbf{r}^{\prime}, 0\right)\right]=\mathbf{c}_{\Pi}\left(\mathbf{r}, \mathbf{r}^{\prime}, \omega\right), \\
\frac{i}{\hbar}\left[\mathbf{J}(\mathbf{r}, \omega), \mathbf{X}\left(\mathbf{r}^{\prime}, 0\right)\right]=\mathbf{c}_{P}\left(\mathbf{r}, \mathbf{r}^{\prime}, \omega\right),
\end{gathered}
$$

and analogous relations for the other coefficients.

It should be noted that the coefficients in Eqs. (53a)-(53d) are all proportional to the imaginary part $\chi_{i}(\mathbf{r}, \omega)$ of the susceptibility. Furthermore, the coefficient in Eq. (53e) is proportional to the bath coupling parameter $v_{\omega}$. In the absence of absorption the dielectric is not coupled to a bath, so that $v_{\omega}$ vanishes. As Eq. (19) shows, the imaginary part of the susceptibility vanishes in that case as well, at least for all frequencies that are off-resonance. Hence, all coefficients (53) are zero in this case, so that the noise-current density itself disappears. Clearly, the present formalism loses its meaning for a nonabsorptive dielectric.

As the noise-current density is fully known now in terms of the canonical variables, we can proceed and derive its properties. This will be the subject in Sec. VI.

\section{PROPERTIES OF THE NOISE-CURRENT DENSITY}

In this section we will determine a few of the properties of the noise-current density $\mathbf{J}(\mathbf{r}, \omega)$. In particular, we will focus on its commutation relations.

We start by considering the commutator of $\mathbf{J}(\mathbf{r}, \omega)$ with the Hamiltonian (3). To evaluate this commutator, we might use the expressions (52)-(53), employ the canonical commutation relations (4) and evaluate all contributions in a systematic way. Owing to the complexity of Eq. (53), this is a rather tedious task. A more convenient way to obtain the commutator is to use the expression (45) for $\mathbf{J}(\mathbf{r}, \omega)$ in terms of $\overline{\mathbf{J}}(\mathbf{r},-i \omega+0)$ and $\breve{\mathbf{J}}(\mathbf{r}, i \omega+0)$. The commutators of the latter with the Hamiltonian can be found without difficulty. In fact, one gets by evaluating the commutators of Eqs. (27) and (33) with (3), or more straightforwardly, by employing the equations of motion (6)

$$
\begin{aligned}
\frac{i}{\hbar}[H, \overline{\mathbf{J}}(\mathbf{r}, p)]= & p \overline{\mathbf{J}}(\mathbf{r}, p)+\frac{1}{\mu_{0} p}(\boldsymbol{\nabla} \times[\boldsymbol{\nabla} \times \mathbf{E}(\mathbf{r}, 0)] \\
& \left.+\frac{p^{2}}{c^{2}} \bar{\varepsilon}(\mathbf{r}, p) \mathbf{E}(\mathbf{r}, 0)\right),
\end{aligned}
$$

and

$$
\begin{aligned}
\frac{i}{\hbar}[H, \breve{\mathbf{J}}(\mathbf{r}, p)]= & -p \breve{\mathbf{J}}(\mathbf{r}, p)+\frac{1}{\mu_{0} p}(\boldsymbol{\nabla} \times[\boldsymbol{\nabla} \times \mathbf{E}(\mathbf{r}, 0)] \\
& \left.+\frac{p^{2}}{c^{2}} \bar{\varepsilon}(\mathbf{r}, p) \mathbf{E}(\mathbf{r}, 0)\right),
\end{aligned}
$$

where the electric field is taken at time $t=0$. Using these expressions, we find as the contribution from the first two terms in Eq. (45) to the commutator $(i / \hbar)[H, \mathbf{J}(\mathbf{r}, \omega)]$,

$$
-\frac{i \omega}{2 \pi}[\overline{\mathbf{J}}(\mathbf{r},-i \omega+0)+\breve{\mathbf{J}}(\mathbf{r}, i \omega+0)]+\frac{\varepsilon_{0}}{\pi} \omega \chi_{i}(\mathbf{r}, \omega) \mathbf{E}(\mathbf{r}, 0) .
$$

Furthermore, the last term in Eq. (45) contributes

$$
\begin{aligned}
& \frac{1}{\pi c^{2}} \omega^{3} \chi_{i}(\mathbf{r}, \omega) \int d \mathbf{r}^{\prime} \overline{\mathbf{G}}\left(\mathbf{r}, \mathbf{r}^{\prime}, i \omega+0\right) \cdot \breve{\mathbf{J}}\left(\mathbf{r}^{\prime}, i \omega+0\right) \\
& -\frac{\varepsilon_{0}}{\pi} \omega \chi_{i}(\mathbf{r}, \omega) \mathbf{E}(\mathbf{r}, 0) .
\end{aligned}
$$

On adding the two contributions, we see that the terms depending on $\mathbf{E}(\mathbf{r}, 0)$ cancel. The remaining terms are proportional to $\mathbf{J}(\mathbf{r}, \omega)$, so that we arrive at the simple result

$$
\frac{i}{\hbar}[H, \mathbf{J}(\mathbf{r}, \omega)]=-i \omega \mathbf{J}(\mathbf{r}, \omega) .
$$

To understand how this commutation property comes about, it is useful to give a somewhat more formal derivation of the commutator. To that end we start by remarking that for an arbitrary operator $\Omega(t)$ the commutator of the Hamiltonian with its Laplace transform $\bar{\Omega}(p)$ follows directly by Laplace-transforming the equation of motion in the Heisenberg picture:

$$
\frac{i}{\hbar}[H, \bar{\Omega}(p)]=p \bar{\Omega}(p)-\Omega(0) .
$$

Writing the analogous equation for the backward Laplace transform,

$$
\frac{i}{\hbar}[H, \breve{\Omega}(p)]=-p \breve{\Omega}(p)+\Omega(0),
$$

and adding the two equations after substitution of the appropriate arguments $p$, we get 


$$
\begin{aligned}
& \frac{i}{\hbar}[H,[\bar{\Omega}(-i \omega+0)+\breve{\Omega}(i \omega+0)]] \\
& \quad=-i \omega[\bar{\Omega}(-i \omega+0)+\breve{\Omega}(i \omega+0)] .
\end{aligned}
$$

In particular, one gets for $\Omega(t)=\mathbf{E}(\mathbf{r}, t)$ by comparison with Eq. (43),

$$
\frac{i}{\hbar}\left[H, \mathbf{E}^{(+)}(\mathbf{r}, \omega)\right]=-i \omega \mathbf{E}^{(+)}(\mathbf{r}, \omega) .
$$

Of course, this could not be otherwise: if it did not hold, the time dependence in Eq. (41) would be compromised. By invoking the definition (44) of the noise-current density in terms of the positive-frequency Fourier component of the electric field, it is immediately clear now that $\mathbf{J}(\mathbf{r}, \omega)$ must satisfy a commutation relation of the same form, which is indeed what we got in Eq. (59).

It should be noted that the commutator expression in Eq. (55) contains an additional term that differs from that in Eq. (60). The reason for this discrepancy is that $\overline{\mathbf{J}}(\mathbf{r}, p)$ has not been defined as the Laplace transform of some operator $\mathbf{J}(\mathbf{r}, t)$, as we noticed already in Sec. III. Similar remarks apply to $\breve{\mathbf{J}}(\mathbf{r}, p)$.

Let us now turn our attention to the commutator of $\mathbf{J}(\mathbf{r}, \omega)$ with its Hermitian conjugate at a different position and frequency. In view of the general form Eq. (45) it is convenient to start by calculating the three commutators involving $\overline{\mathbf{J}}$ and $\breve{\mathbf{J}}$. These follow by substitution of Eqs. (27) and (33) and use of the canonical commutation relations (4). The results are given in Eqs. (B1) and (B3) of Appendix B. As shown there, these commutators of $\overline{\mathbf{J}}$ and $\breve{\mathbf{J}}$ can be used to prove the commutation relation

$$
\left[\mathbf{J}(\mathbf{r}, \omega),\left[\mathbf{J}\left(\mathbf{r}^{\prime}, \omega^{\prime}\right)\right]^{\dagger}\right]=\frac{\varepsilon_{0} \hbar}{\pi} \omega^{2} \chi_{i}(\mathbf{r}, \omega) \delta\left(\omega-\omega^{\prime}\right) \mathbf{I} \delta\left(\mathbf{r}-\mathbf{r}^{\prime}\right) .
$$

In an analogous fashion one may evaluate the commutator of the noise-current density with its counterpart for different arguments. It is found that this commutator vanishes:

$$
\left[\mathbf{J}(\mathbf{r}, \omega), \mathbf{J}\left(\mathbf{r}^{\prime}, \omega^{\prime}\right)\right]=0 .
$$

As shown in Appendix B, the commutators (64) and (65) appear as the results of calculations in which several terms cancel one another. In fact, all nonlocal terms involving transverse delta functions and Green functions drop out. The final answers show that $\mathbf{J}(\mathbf{r}, \omega)$ is a strictly local operator in its space variable: for all $\mathbf{r}^{\prime} \neq \mathbf{r}$ it commutes both with $\mathbf{J}\left(\mathbf{r}^{\prime}, \omega\right)$ and with the Hermitian conjugate of the latter. Moreover, the right-hand sides of Eqs. (64) and (65) show that the noise-current density is local in the frequency as well: the commutators vanish for $\omega \neq \omega^{\prime}$. As a final comment we note that the commutator Eq. (64) is proportional to the imaginary part of the susceptibility. Hence, it vanishes if there is no absorption. As we have seen above, the noisecurrent density itself vanishes in that case, so that Eq. (64) becomes a trivial identity.
As demonstrated above, the commutator properties in Eqs. (59), (64), and (65) follow from the dynamics of the damped-polariton model. These commutation relations are the same as the postulated relations of the noise-current density in the phenomenological quantization scheme [17-23]. Evidently, the status of the commutation relations is rather different in both schemes. Our results provide a justification for the postulates in the phenomenological theory.

The collection of operators $\mathbf{J}(\mathbf{r}, \omega)$ possesses another convenient property: together with their Hermitian conjugates they form a complete basis set for the canonical variables of the model. It means that each of these variables can be written as a linear combination of the operators from the basis. To prove this statement, we may argue as follows. Let us tentatively write the vector potential as

$$
\mathbf{A}(\mathbf{r}, 0)=\int d \mathbf{r}^{\prime} \int_{0}^{\infty} d \omega \mathbf{J}\left(\mathbf{r}^{\prime}, \omega\right) \cdot \mathbf{f}_{A}\left(\mathbf{r}^{\prime}, \mathbf{r}, \omega\right)+\text { H.c. },
$$

with as yet unknown tensorial coefficients $\mathbf{f}_{A}$. Taking the commutator of both sides with $\left[\mathbf{J}\left(\mathbf{r}^{\prime \prime}, \omega^{\prime}\right)\right]^{\dagger}$ we find from Eqs. (54b), (64), and (65):

$$
\mathbf{f}_{A}\left(\mathbf{r}, \mathbf{r}^{\prime}, \omega\right)=\frac{i \pi}{\varepsilon_{0} \omega^{2} \chi_{i}(\mathbf{r}, \omega)} \mathbf{c}_{\mathbf{I}}^{*}\left(\mathbf{r}, \mathbf{r}^{\prime}, \omega\right),
$$

so that we get:

$$
\begin{aligned}
\mathbf{A}(\mathbf{r}, 0)= & \frac{i \pi}{\varepsilon_{0}} \int d \mathbf{r}^{\prime} \int_{0}^{\infty} d \omega \frac{1}{\omega^{2} \chi_{i}\left(\mathbf{r}^{\prime}, \omega\right)} \\
& \times \mathbf{J}\left(\mathbf{r}^{\prime}, \omega\right) \cdot \mathbf{c}_{\boldsymbol{\Pi}}^{*}\left(\mathbf{r}^{\prime}, \mathbf{r}, \omega\right)+\text { H.c. }
\end{aligned}
$$

To really establish the validity of this equality, which we found by merely assuming the general form in Eq. (66), we insert Eq. (52) on the right-hand side, which then becomes a linear combination of the canonical variables. Upon evaluating the resulting integrals with the techniques of Appendices $A$ and $B$, we indeed find that only the term with the vector potential survives, and that the left-hand side is recovered.

Two other examples of identities, which may be checked in an analogous way, are

$$
\begin{aligned}
\Pi(\mathbf{r}, 0)= & -\frac{i \pi}{\varepsilon_{0}} \int d \mathbf{r}^{\prime} \int_{0}^{\infty} d \omega \frac{1}{\omega^{2} \chi_{i}\left(\mathbf{r}^{\prime}, \omega\right)} \mathbf{J}\left(\mathbf{r}^{\prime}, \omega\right) \cdot \mathbf{c}_{A}^{*}\left(\mathbf{r}^{\prime}, \mathbf{r}, \omega\right) \\
& + \text { H.c., } \\
\mathbf{X}(\mathbf{r}, 0)= & \frac{i \pi}{\varepsilon_{0}} \int d \mathbf{r}^{\prime} \int_{0}^{\infty} d \omega \frac{1}{\omega^{2} \chi_{i}\left(\mathbf{r}^{\prime}, \omega\right)} \mathbf{J}\left(\mathbf{r}^{\prime}, \omega\right) \cdot \mathbf{c}_{P}^{*}\left(\mathbf{r}^{\prime}, \mathbf{r}, \omega\right) \\
& + \text { H.c. }
\end{aligned}
$$

Similar identities are found to be valid for the canonical variables $\mathbf{P}, \mathbf{Y}_{\omega}$, and $\mathbf{Q}_{\omega}$. Since all canonical variables can thus be expressed in terms of $\mathbf{J}$ and its Hermitian conjugate, these operators must form a complete basis, as we set out to prove.

The completeness of the set of operators $\mathbf{J}(\mathbf{r}, \omega)$ and $[\mathbf{J}(\mathbf{r}, \omega)]^{\dagger}$, and their properties Eqs. (59), (64), and (65) im- 
ply that the noise-current density is proportional to the diagonalizing operator of the system Hamiltonian (3). In fact, we may write:

$$
H=\frac{\pi}{\varepsilon_{0}} \int d \mathbf{r} \int_{0}^{\infty} d \omega \frac{1}{\omega \chi_{i}(\mathbf{r}, \omega)}[\mathbf{J}(\mathbf{r}, \omega)]^{\dagger} \cdot \mathbf{J}(\mathbf{r}, \omega) .
$$

We have checked that Eq. (3) can be recovered from Eq. (70). This is accomplished by substituting Eq. (52) with (53) in (70) and evaluating the resulting expression in terms of the canonical variables. A few details of this calculation are given in Appendix C. It should be noted that the two expressions for the Hamiltonian do not agree completely: they differ by a $c$-number, which corresponds to a zero-point energy.

Now that we have succeeded in obtaining the diagonalizing operators of our model, we can determine the full time dependence of the vector potential, the electric field, the polarization density, or any of the dynamic variables that we have considered above. For example, the vector potential at time $t$ follows from Eq. (68) by substituting the timedependent noise-current density $e^{-i \omega t} \mathbf{J}\left(\mathbf{r}^{\prime}, \omega\right)$ in the integrand. Inserting the expression (53b) for $\mathbf{c}_{\Pi}$, and using Eq. (35) we get

$\mathbf{A}(\mathbf{r}, t)=-\mu_{0} \int d \mathbf{r}^{\prime} \int_{0}^{\infty} d \omega e^{-i \omega t} \mathbf{G}_{\mathrm{T}}\left(\mathbf{r}, \mathbf{r}^{\prime}, \omega\right) \cdot \mathbf{J}\left(\mathbf{r}^{\prime}, \omega\right)+$ H.c.

In the phenomenological quantization scheme an integral representation of the same form shows up [22]. However, in that theory the noise-current density $\mathbf{J}(\mathbf{r}, \omega)$ is a formal operator. In the present model we have an explicit expression for $\mathbf{J}$ at our disposal. In fact, by substituting Eq. (52) we may evaluate the right-hand side of Eq. (71) in terms of the canonical variables at $t=0$. The results are presented in Appendix D. As shown there, the vector potential gets a simple form when enough time has passed for transients to die out. In that long-time limit, it reduces to a linear combination of bath operators only:

$$
\begin{aligned}
\mathbf{A}(\mathbf{r}, t) \simeq & \frac{1}{2 c^{2}} \int d \mathbf{r}^{\prime} \frac{1}{\alpha^{\prime}} \int_{0}^{\infty} d \omega e^{-i \omega t} v_{\omega}^{\prime} \chi\left(\mathbf{r}^{\prime}, \omega\right) \\
& \times \mathbf{G}_{\mathrm{T}}\left(\mathbf{r}, \mathbf{r}^{\prime}, \omega\right) \cdot\left[\omega^{2} \mathbf{Y}_{\omega}\left(\mathbf{r}^{\prime}, 0\right)+\frac{i \omega}{\rho^{\prime}} \mathbf{Q}_{\omega}\left(\mathbf{r}^{\prime}, 0\right)\right] \\
& + \text { H.c. }
\end{aligned}
$$

This expression has the same form as Eq. (71), with the noise-current operator replaced by:

$$
\mathbf{J}_{l}(\mathbf{r}, \omega)=-\frac{\varepsilon_{0}}{2 \alpha} v_{\omega} \chi(\mathbf{r}, \omega)\left[\omega^{2} \mathbf{Y}_{\omega}(\mathbf{r}, 0)+\frac{i \omega}{\rho} \mathbf{Q}_{\omega}(\mathbf{r}, 0)\right] .
$$

The combination between square brackets is proportional to the annihilation operator of the bath harmonic oscillators at the chosen position and frequency. Indeed, $\mathbf{J}_{l}$ satisfies the same standard commutation relations (64)-(65) as J. The vector potential thus depends on the bath annihilation and creation operators only, when all transients have died out.
This result for the long-time limit is the generalization of a similar finding for the homogeneous damped-polariton model, which we already discussed [15].

The time-dependent electric field $\mathbf{E}(\mathbf{r}, t)$ could be found in principle by separately evaluating its transverse part $-\boldsymbol{\Pi}(\mathbf{r}, t) / \varepsilon_{0}$ from Eq. (69a) and its longitudinal part $[\alpha \mathbf{X}(\mathbf{r}, t)]_{\mathrm{L}} / \varepsilon_{0}$ from Eq. (69b), and adding the two contributions. However, a simpler way to obtain $\mathbf{E}(\mathbf{r}, t)$ is to insert the solution of Eq. (44) into the general form Eq. (41). In this way we get

$$
\begin{aligned}
\mathbf{E}(\mathbf{r}, t)= & -i \mu_{0} \int d \mathbf{r}^{\prime} \int_{0}^{\infty} d \omega e^{-i \omega t} \omega \mathbf{G}\left(\mathbf{r}, \mathbf{r}^{\prime}, \omega\right) \cdot \mathbf{J}\left(\mathbf{r}^{\prime}, \omega\right) \\
& + \text { H.c. }
\end{aligned}
$$

In Appendix D, it is shown that in the long-time limit the electric field is given by an expression of the same form as Eq. (74), with $\mathbf{J}$ replaced by $\mathbf{J}_{l}$. By using the commutation relations (64)-(65), which are valid for $\mathbf{J}_{l}$ as well, one may show that the commutator of the electric field and the vector potential in the long-time limit has the standard form as Eq. (5a).

As a final example, we consider the time-dependent polarization density $-\alpha \mathbf{X}(\mathbf{r}, t)$. It follows from Eq. (69b) as:

$$
\begin{aligned}
-\alpha \mathbf{X}(\mathbf{r}, t)= & -\frac{i \pi \alpha}{\varepsilon_{0}} \int d \mathbf{r}^{\prime} \int_{0}^{\infty} d \omega \frac{1}{\omega^{2} \chi_{i}\left(\mathbf{r}^{\prime}, \omega\right)} e^{-i \omega t} \\
& \times \mathbf{J}\left(\mathbf{r}^{\prime}, \omega\right) \cdot \mathbf{c}_{P}^{*}\left(\mathbf{r}^{\prime}, \mathbf{r}, \omega\right)+\text { H.c. }
\end{aligned}
$$

Substituting Eq. (53d) we get

$$
\begin{aligned}
-\alpha \mathbf{X}(\mathbf{r}, t)= & -\frac{i}{c^{2}} \int d \mathbf{r}^{\prime} \int_{0}^{\infty} d \omega e^{-i \omega t} \omega \chi(\mathbf{r}, \omega) \\
& \times \mathbf{G}\left(\mathbf{r}, \mathbf{r}^{\prime}, \omega\right) \cdot \mathbf{J}\left(\mathbf{r}^{\prime}, \omega\right) \\
& +i \int_{0}^{\infty} d \omega e^{-i \omega t} \frac{1}{\omega} \mathbf{J}(\mathbf{r}, \omega)+\text { H.c. },
\end{aligned}
$$

where we used Eq. (35). This form for the time-dependent polarization density shows that it is the sum of a term involving the properties of the medium through the susceptibility and a term which is determined by the noise-current density only. In fact, this is consistent with Eq. (49), which was written in terms of the positive-frequency Fourier components. Indeed, the integrand in the first term on the righthand side of Eq. (76) is proportional to the positivefrequency part of the electric field, as we have seen in Eq. (74). In Appendix D the time-dependent polarization density is evaluated in terms of the canonical variables at $t=0$. Furthermore, it is shown there that the long-time limit of $-\alpha \mathbf{X}(\mathbf{r}, t)$ follows from Eq. (76) by replacing $\mathbf{J}$ by $\mathbf{J}_{l}$, as was found above for the vector potential and the electric field.

The expressions (71), (74), and (76) give the complete time dependence of the vector potential, the electric field and the polarization density in the inhomogeneous dampedpolariton model. For the special case of a homogeneous medium, the expressions reduce to those given in Ref. [9]. As we have seen, the implicit dependence on the canonical vari- 
ables at $t=0$ can be made explicit by substitution of the noise-current density in the form of Eq. (52).

Now that we have found in Eqs. (71) and (74) the explicit time dependence of the field operators $\mathbf{A}$ and $\mathbf{E}$ in terms of the noise-current density $\mathbf{J}$, we can determine the commutators $\left[\mathbf{E}(\mathbf{r}, t), \mathbf{A}\left(\mathbf{r}^{\prime}, t\right)\right]$ and $\left[\mathbf{E}(\mathbf{r}, t), \mathbf{B}\left(\mathbf{r}^{\prime}, t\right)\right]$ for arbitrary $t$. Actually, for $t=0$ we have already determined these in Eq. (5) and the fact that these commutators are mediumindependent almost directly followed from the standard commutation relations (4). Now that we have integrated out the dynamics of the material variables, the expressions Eq. (71) and (74) clearly both depend on the medium through the Green function $\mathbf{G}\left(\mathbf{r}, \mathbf{r}^{\prime}, \omega\right)$ and the noise-current density $\mathbf{J}\left(\mathbf{r}^{\prime}, \omega\right)$. However, since no approximations were made in order to obtain the time dependence of the field operators, their commutators should still be medium independent, and equal to those at $t=0$. With the use of Eqs. (34)-(36) and the Green-function sum rule (A4) one can verify that the commutators $\left[\mathbf{E}(\mathbf{r}, t), \mathbf{A}\left(\mathbf{r}^{\prime}, t\right)\right]$ and $\left[\mathbf{E}(\mathbf{r}, t), \mathbf{B}\left(\mathbf{r}^{\prime}, t\right)\right]$ indeed have the medium independent values of Eq. (5). A medium independent commutator $\left[\mathbf{E}(\mathbf{r}, t), \mathbf{B}\left(\mathbf{r}^{\prime}, t\right)\right]$ was also found in the phenomenological scheme [23], which was the principal argument in showing that the phenomenological scheme is consistent with (although not founded on) quantum electrodynamics. Finally, it may be remarked that in our theory the commutators $\left[\mathbf{P}(\mathbf{r}, t), \mathbf{X}\left(\mathbf{r}^{\prime}, t\right)\right]$ and $\left[\mathbf{Q}_{\omega}(\mathbf{r}, t), \mathbf{Y}_{\omega^{\prime}}\left(\mathbf{r}^{\prime}, t\right)\right]$ are also medium independent; these commutators have no counterparts in the phenomenological theory.

\section{CONCLUSION AND DISCUSSION}

By solving the inhomogeneous damped-polariton model we have established a rigorous basis for the phenomenological quantization procedure, which has been used to describe quantum phenomena in linear lossy dielectrics with great success. Up to now such a firm basis was available for homogeneous dielectrics only, through the pioneering work of Huttner and Barnett $[8,9]$.

As a tool in our treatment we have used forward and backward Laplace transformations. With the help of these we solved the equations of motion for the canonical variables. The Laplace transforms of the electric field were shown to obey wave equations with source terms that could be expressed in terms of the canonical variables at time $t=0$. Upon introducing the Green function of these wave equations we were able to derive an expression for the positive-frequency Fourier component of the time-dependent electric field. The latter was found to satisfy a wave equation with a frequencydependent source term that could be interpreted as a noisecurrent density for the inhomogeneous damped-polariton model. Explicit expressions for this noise-current density in terms of the canonical variables of the system have been derived. By establishing its algebraic properties we could prove that it is proportional to the diagonalizing operator of the model. Once we have shown this, the time-dependence of all relevant operators can be determined. As an illustration we gave the time-dependent expressions for the vector potential, the electric field, and the polarization density.
In order to show the internal consistency of our results, we have derived and employed several frequency sum rules for the tensorial Green function and for the susceptibility, namely Eqs. (A4), (A5), (C6), (C8), and (C9). The outcomes solely depend on the high-frequency asymptotic behavior of the Green function and the susceptibility. In the present model this behavior is determined by the values of the (local) parameters $\alpha, \rho, \omega_{0}$, and $v_{\omega}$. The Green function sum rules are generalizations of velocity sum rules that have been derived for homogeneous dielectrics $[9,15]$.

We have expressed all field operators in terms of the noise-current density operators $\mathbf{J}(\mathbf{r}, \omega)$, which also diagonalize the Hamiltonian. These operators and their Hermitian conjugates were proved to be local both in position and in frequency: any pair of them commute when taken at different positions and/or different frequencies. The locality in position is not self-evident $a$ priori, as some of the canonical variables of the model, namely $\mathbf{A}$ and $\mathbf{\Pi}$, satisfy a commutation relation (4a) with a nonlocal transverse delta function. The positive-frequency Fourier component $\mathbf{E}^{(+)}(\mathbf{r}, \omega)$ of the electric field is nonlocal in space as well: it does not commute with its Hermitian conjugate at a position $\mathbf{r}^{\prime}$ (and at the same frequency $\omega)$. The locality of $\mathbf{J}(\mathbf{r}, \omega)$ with respect to the frequency is connected to the validity of a generalized optical theorem (B7) for the Green function. When the independent frequency variables in this theorem are chosen to be equal, it reduces to the standard form of the optical theorem [22].

The diagonalizing operators are not unique. For example, if one breaks up the noise-current density in terms of its canonical elements according to Eq. (52), then for long times after the initial time $t=0$ one finds that the field operators are determined only by the initial bath operators, since timedependent coefficients of other canonical variables all decay exponentially fast. If only long times are considered, $\mathbf{J}(\mathbf{r}, \omega)$ can be taken to be proportional to the initial annihilation operator of the bath harmonic oscillator at position $\mathbf{r}$ and frequency $\omega$. We stressed this point in Ref. [15] for homogeneous dielectrics. Other diagonalizing operators can be constructed by transforming the noise-current density $\mathbf{J}(\mathbf{r}, \omega)$ with arbitrary unitary transformations $\mathbf{U}\left(\mathbf{r}, \mathbf{r}^{\prime}, \omega\right)$, but these would not have the physical interpretation of noise-current density operators.

Our solution provides detailed information on the dynamical behavior of absorptive dielectrics. This information can be used to study dynamical processes like spontaneous emission of guest atoms in inhomogeneous media. For instance, transient effects in emission processes, which we studied in homogeneous media [15], can now be investigated in the general inhomogeneous case. Local-field effects, which by their very nature are brought about by inhomogeneities in the medium, form another field of interest for which our solution may be helpful.

To obtain our results we have employed a Laplacetransform technique which we used before [15]. An alternative method, which was adopted in Ref. [9], is based on a diagonalization procedure due to Fano [40]. We have been able to carry out the diagonalization of the inhomogeneous damped-polariton model along those lines as well. Details of that work will be published elsewhere [41]. 


\section{ACKNOWLEDGMENT}

The authors would like to thank Dr. A.J. van Wonderen for many stimulating discussions.

\section{APPENDIX A: SHORT-TIME LIMIT OF THE ELECTRIC FIELD}

In Sec. IV the electric-field operator $\mathbf{E}(\mathbf{r}, t)$ has been found as a Fourier integral (41), with the positive-frequency Fourier component $\mathbf{E}^{(+)}(\mathbf{r}, \omega)$ given in Eq. (42). It contains the source terms $\overline{\mathbf{J}}$ and $\breve{\mathbf{J}}$, which have been given in Eqs. (27) and (33) as linear combinations of the canonical variables at $t=0$. As a check, we shall verify that the Fourier integral in Eq. (41) reduces to $\mathbf{E}(\mathbf{r}, 0)$ in the limit $t \rightarrow 0$. As we shall see, the proof will depend on the validity of a few sum rules for the Green function.

By employing the identity $[\overline{\mathbf{E}}(\mathbf{r},-i \omega+0)]^{\dagger}=\overline{\mathbf{E}}(\mathbf{r}, i \omega+0)$, and the corresponding identity for the backward Laplace transform, one may write the Fourier integral representing $\mathbf{E}(\mathbf{r}, 0)$ as

$$
\frac{1}{2 \pi} \int_{-\infty}^{\infty} d \omega[\overline{\mathbf{E}}(\mathbf{r},-i \omega+0)+\breve{\mathbf{E}}(\mathbf{r},-i \omega+0)]
$$

Upon substituting Eqs. (37), (38), (27), and (33), we find that all terms with $\mathbf{A}\left(\mathbf{r}^{\prime}, 0\right), \mathbf{P}\left(\mathbf{r}^{\prime}, 0\right)$, and $\mathbf{Y}_{\omega^{\prime}}\left(\mathbf{r}^{\prime}, 0\right)$ cancel. We are left with the following expression:

$$
\begin{aligned}
& -\frac{i}{\pi} \int_{-\infty}^{\infty} d \omega \int d \mathbf{r}^{\prime} \overline{\mathbf{G}}\left(\mathbf{r}, \mathbf{r}^{\prime},-i \omega+0\right) \cdot\left\{\mu_{0} \omega \boldsymbol{\Pi}\left(\mathbf{r}^{\prime}, 0\right)\right. \\
& \quad+\mu_{0} \omega\left[\alpha^{\prime} \mathbf{X}\left(\mathbf{r}^{\prime}, 0\right)\right]_{\mathrm{T}^{\prime}}+\frac{\rho^{\prime}}{c^{2} \alpha^{\prime}} \omega^{3} \bar{\chi}\left(\mathbf{r}^{\prime},-i \omega+0\right) \mathbf{X}\left(\mathbf{r}^{\prime}, 0\right) \\
& -\frac{1}{c^{2} \alpha^{\prime} \rho^{\prime}} \omega^{3} \bar{\chi}\left(\mathbf{r}^{\prime},-i \omega+0\right) \\
& \left.\quad \times \int_{0}^{\infty} d \omega^{\prime} \frac{v_{\omega^{\prime}}^{\prime}}{\omega^{\prime 2}-(\omega+i 0)^{2}} \mathbf{Q}_{\omega^{\prime}}\left(\mathbf{r}^{\prime}, 0\right)\right\}
\end{aligned}
$$

This result can be simplified by considering the integrals over $\omega$. The terms with $\boldsymbol{\Pi}$ and $[\alpha \mathbf{X}]_{\mathrm{T}}$ contain the integral $\int_{-\infty}^{\infty} d \omega \omega \overline{\mathbf{G}}\left(\mathbf{r}, \mathbf{r}^{\prime},-i \omega+0\right)$. Since the Green function is analytic for $\omega$ in the upper half-plane, we may evaluate the integral by closing the contour in this half-plane. The Green function satisfies the differential Eq. (34). Now $\bar{\varepsilon}(\mathbf{r}, p)=1$ $+\bar{\chi}(\mathbf{r}, p)$ tends to 1 for large $p$ in the right half-plane, as follows from the expression (19) for $\bar{\chi}$. Hence, the asymptotic form of the Green function for large $\omega$ in the upper half-plane has the same form as the free-space Green function, namely,

$$
\overline{\mathbf{G}}\left(\mathbf{r}, \mathbf{r}^{\prime},-i \omega+0\right) \simeq \frac{c^{2}}{(\omega+i 0)^{2}} \mathbf{I} \delta\left(\mathbf{r}-\mathbf{r}^{\prime}\right)
$$

As a consequence, closing the contour yields the identity

$$
\int_{-\infty}^{\infty} d \omega \omega \overline{\mathbf{G}}\left(\mathbf{r}, \mathbf{r}^{\prime},-i \omega+0\right)=-i \pi c^{2} \mathbf{I} \delta\left(\mathbf{r}-\mathbf{r}^{\prime}\right),
$$

which is in fact a sum rule for the Green function [23].

Likewise, one proves

$$
\begin{aligned}
\int_{-\infty}^{\infty} & d \omega \omega^{3} \overline{\mathbf{G}}\left(\mathbf{r}, \mathbf{r}^{\prime},-i \omega+0\right) \bar{\chi}\left(\mathbf{r}^{\prime},-i \omega+0\right) \\
= & \frac{i \pi c^{2} \alpha^{2}}{\varepsilon_{0} \rho} \mathbf{I} \delta\left(\mathbf{r}-\mathbf{r}^{\prime}\right)
\end{aligned}
$$

since the susceptibility $\bar{\chi}(\mathbf{r},-i \omega+0)$ behaves like $-\left[\alpha^{2} /\left(\varepsilon_{0} \rho\right)\right] /(\omega+i 0)^{2}$ for large $\omega$ in the upper half-plane.

Finally, the contribution of $\mathbf{Q}_{\omega}$ is determined by the integral

$$
\int_{-\infty}^{\infty} d \omega \omega^{3} \overline{\mathbf{G}}\left(\mathbf{r}, \mathbf{r}^{\prime},-i \omega+0\right) \bar{\chi}\left(\mathbf{r}^{\prime},-i \omega+0\right) \frac{1}{\omega^{\prime 2}-(\omega+i 0)^{2}} .
$$

As the integrand is proportional to $(\omega+i 0)^{-3}$ for large $\omega$ in the upper half-plane, a contour deformation leads to a vanishing result.

From the above we conclude that the expression (A2) is equal to

$$
-\frac{1}{\varepsilon_{0}} \boldsymbol{\Pi}(\mathbf{r}, 0)+\frac{1}{\varepsilon_{0}}[\alpha \mathbf{X}(\mathbf{r}, 0)]_{\mathrm{L}},
$$

which is in agreement with Eq. (25).

\section{APPENDIX B: COMMUTATORS OF THE NOISE-CURRENT DENSITY}

In this appendix we derive the commutators of the noisecurrent density with itself and with its Hermitian conjugate. We start from Eq. (45), in which $\mathbf{J}(\mathbf{r}, \omega)$ is given as a linear combination of the source terms $\overline{\mathbf{J}}(\mathbf{r}, p)$ and $\breve{\mathbf{J}}(\mathbf{r}, p)$. From the definition (27) and the canonical commutation relations (4) we obtain the commutator $\left[\overline{\mathbf{J}}, \overline{\mathbf{J}}^{\dagger}\right]$ as

$$
\begin{aligned}
{\left[\overline{\mathbf{J}}(\mathbf{r}, p),\left[\overline{\mathbf{J}}\left(\mathbf{r}^{\prime}, p^{\prime}\right)\right]^{\dagger}\right] } & \\
= & \frac{i \hbar}{\mu_{0}} \frac{p-p^{\prime *}}{p p^{\prime *}}(\nabla \nabla-\mathbf{I} \Delta) \delta\left(\mathbf{r}-\mathbf{r}^{\prime}\right) \\
& -i \varepsilon_{0} \hbar \frac{p p^{\prime *}}{p+p^{\prime *}}\left[\bar{\chi}(\mathbf{r}, p)-\bar{\chi}\left(\mathbf{r}, p^{\prime *}\right)\right] \mathbf{I} \delta\left(\mathbf{r}-\mathbf{r}^{\prime}\right),
\end{aligned}
$$

where we used the auxiliary relation

$$
\begin{aligned}
& \int_{0}^{\infty} d \omega \frac{\omega^{2} v_{\omega}^{2}}{\left(p^{2}+\omega^{2}\right)\left(p^{\prime 2}+\omega^{2}\right)} \\
& \quad=-\rho^{2}+\frac{\alpha^{2} \rho}{\varepsilon_{0}} \frac{1}{p^{2}-p^{\prime 2}}\left[\frac{1}{\bar{\chi}(\mathbf{r}, p)}-\frac{1}{\bar{\chi}\left(\mathbf{r}, p^{\prime}\right)}\right],
\end{aligned}
$$

where for brevity we did not write the position dependence 
of $v_{\omega}, \rho$, and $\alpha$. The commutator $\left[\breve{\mathbf{J}}(\mathbf{r}, p),\left[\breve{\mathbf{J}}\left(\mathbf{r}^{\prime}, p^{\prime}\right)\right]^{\dagger}\right]$ is equal to Eq. (B1), apart from an overall minus sign. Finally, the commutation relation of $\overline{\mathbf{J}}$ with $\breve{\mathbf{J}}^{\dagger}$ is found to be

$$
\begin{aligned}
& {\left[\overline{\mathbf{J}}(\mathbf{r}, p),\left[\breve{\mathbf{J}}\left(\mathbf{r}^{\prime}, p^{\prime}\right)\right]^{\dagger}\right]} \\
& =\frac{i \hbar}{\mu_{0}} \frac{p+p^{\prime *}}{p p^{\prime *}}(\nabla \nabla-\mathbf{I} \Delta) \delta\left(\mathbf{r}-\mathbf{r}^{\prime}\right) \\
& \quad-i \varepsilon_{0} \hbar \frac{p p^{\prime *}}{p-p^{\prime *}}\left[\bar{\chi}(\mathbf{r}, p)-\bar{\chi}\left(\mathbf{r}, p^{\prime *}\right)\right] \mathbf{I} \delta\left(\mathbf{r}-\mathbf{r}^{\prime}\right) .
\end{aligned}
$$

The last term on the right-hand side is not singular for $p$ $=p^{\prime *}$. In fact, it is proportional to the derivative of the susceptibility in that case. It should be noted that the commutators (B1) and (B3) are local, as they vanish for $\mathbf{r} \neq \mathbf{r}^{\prime}$. This is not self-evident, since the definitions (27) and (33) contain nonlocal longitudinal terms. When evaluating the commutators, one finds that the nonlocal transverse delta function in the canonical commutator (4a) compensates for the nonlocal terms in $\overline{\mathbf{J}}$ and $\breve{\mathbf{J}}$.

Having derived the commutators for $\overline{\mathbf{J}}$ and $\breve{\mathbf{J}}$, we can determine the commutator of $\mathbf{J}(\mathbf{r}, \omega)$ with $\left[\mathbf{J}\left(\mathbf{r}^{\prime}, \omega^{\prime}\right)\right]^{\dagger}$, by using Eq. (45). In $\overline{\mathbf{J}}$ we have to substitute $p=-i \omega+0$ or $p^{\prime}=$ $-i \omega^{\prime}+0$, while in $\breve{\mathbf{J}}$ the argument is $p=i \omega+0$ or $p^{\prime}=i \omega^{\prime}+0$. Sorting out the various terms, we get three different types of contributions, namely those containing either no Greenfunction factor, or one or two such factors. In the first contribution the terms proportional to $(\nabla \nabla-\mathbf{I} \Delta)$ drop out. The terms proportional to I $\delta\left(\mathbf{r}-\mathbf{r}^{\prime}\right)$ cancel as well, unless $\omega$ and $\omega^{\prime}$ are equal:

$$
\begin{aligned}
\frac{1}{4 \pi^{2}}[ & \overline{\mathbf{J}}(\mathbf{r},-i \omega+0)+\breve{\mathbf{J}}(\mathbf{r}, i \omega+0),\left[\overline{\mathbf{J}}\left(\mathbf{r}^{\prime},-i \omega^{\prime}+0\right)\right]^{\dagger} \\
& \left.+\left[\breve{\mathbf{J}}\left(\mathbf{r}^{\prime}, i \omega^{\prime}+0\right)\right]^{\dagger}\right] \\
& =\frac{\varepsilon_{0} \hbar}{\pi} \omega^{2} \chi_{i}(\mathbf{r}, \omega) \delta\left(\omega-\omega^{\prime}\right) \mathbf{I} \delta\left(\mathbf{r}-\mathbf{r}^{\prime}\right) .
\end{aligned}
$$

The contribution with a single Green-function factor is

$\frac{\varepsilon_{0} \hbar}{\pi^{2} c^{2}} \frac{\omega \omega^{\prime}}{\omega-\omega^{\prime}-i 0} \chi_{i}(\mathbf{r}, \omega) \chi_{i}\left(\mathbf{r}^{\prime}, \omega^{\prime}\right)\left[\omega^{2} \overline{\mathbf{G}}\left(\mathbf{r}, \mathbf{r}^{\prime}, i \omega+0\right)\right.$

$\left.-\omega^{\prime 2} \overline{\mathbf{G}}\left(\mathbf{r}, \mathbf{r}^{\prime},-i \omega^{\prime}+0\right)\right]$.

Finally, the contribution with two Green-function factors is found as

$$
\begin{aligned}
\frac{\varepsilon_{0} \hbar}{\pi^{2} c^{2}} & \omega \omega^{\prime}\left(\omega+\omega^{\prime}\right) \chi_{i}(\mathbf{r}, \omega) \chi_{i}\left(\mathbf{r}^{\prime}, \omega^{\prime}\right) \int d \mathbf{r}^{\prime \prime} \overline{\mathbf{G}}\left(\mathbf{r}, \mathbf{r}^{\prime \prime}, i \omega+0\right) \\
& \cdot\left\{\boldsymbol{\nabla}^{\prime \prime} \times\left[\nabla^{\prime \prime} \times \overline{\mathbf{G}}\left(\mathbf{r}^{\prime \prime}, \mathbf{r}^{\prime},-i \omega^{\prime}+0\right)\right]\right\} \\
& +\frac{\varepsilon_{0} \hbar}{\pi^{2} c^{4}} \frac{\omega^{3} \omega^{\prime 3}}{\omega-\omega^{\prime}-i 0} \chi_{i}(\mathbf{r}, \omega) \chi_{i}\left(\mathbf{r}^{\prime}, \omega^{\prime}\right) \int d \mathbf{r}^{\prime \prime}\left[\bar{\chi}\left(\mathbf{r}^{\prime \prime}, i \omega+0\right)\right. \\
- & \left.\bar{\chi}\left(\mathbf{r}^{\prime \prime},-i \omega^{\prime}+0\right)\right] \overline{\mathbf{G}}\left(\mathbf{r}, \mathbf{r}^{\prime \prime}, i \omega+0\right) \cdot \overline{\mathbf{G}}\left(\mathbf{r}^{\prime \prime}, \mathbf{r}^{\prime},-i \omega^{\prime}+0\right)
\end{aligned}
$$

The second integral appearing here can be split into two parts, which may be rewritten with the use of Eq. (34) and (36). After a partial integration we arrive at the identity

$$
\begin{aligned}
& \int d \mathbf{r}^{\prime \prime}\left[\bar{\chi}\left(\mathbf{r}^{\prime \prime}, i \omega+0\right)-\bar{\chi}\left(\mathbf{r}^{\prime \prime},-i \omega^{\prime}+0\right)\right] \overline{\mathbf{G}}\left(\mathbf{r}, \mathbf{r}^{\prime \prime}, i \omega+0\right) \cdot \overline{\mathbf{G}}\left(\mathbf{r}^{\prime \prime}, \mathbf{r}^{\prime},-i \omega^{\prime}+0\right) \\
& =-\frac{c^{2}}{\omega^{2} \omega^{\prime 2}}\left[\omega^{2} \overline{\mathbf{G}}\left(\mathbf{r}, \mathbf{r}^{\prime}, i \omega+0\right)-\omega^{\prime 2} \overline{\mathbf{G}}\left(\mathbf{r}, \mathbf{r}^{\prime},-i \omega^{\prime}+0\right)+\left(\omega^{2}-\omega^{\prime 2}\right) \int d \mathbf{r}^{\prime \prime} \overline{\mathbf{G}}\left(\mathbf{r}, \mathbf{r}^{\prime \prime}, i \omega+0\right) \cdot\left\{\nabla^{\prime \prime}\left[\nabla^{\prime \prime} \times \overline{\mathbf{G}}\left(\mathbf{r}^{\prime \prime}, \mathbf{r}^{\prime},-i \omega^{\prime}+0\right)\right]\right\}\right] .
\end{aligned}
$$

For arbitrary $\omega$ and $\omega^{\prime}$ this identity has the form of a generalized optical theorem for the Green function. By putting $\omega$ $=\omega^{\prime}$ one recovers the optical theorem that has been discussed before [22]. When Eq. (B7) is used in Eq. (B6), it turns out that all terms with spatial derivatives cancel, while the remaining terms are the opposite of Eq. (B5). As a consequence, we are left with Eq. (B4), so that we have proven the commutation relation (64).

The commutator of $\mathbf{J}(\mathbf{r}, \omega)$ with $\mathbf{J}\left(\mathbf{r}^{\prime}, \omega^{\prime}\right)$ can be evaluated in a similar way. As a preparation, one needs the com- mutators of $\overline{\mathbf{J}}$ and $\breve{\mathbf{J}}$ with their counterparts for different arguments. These operators satisfy the identities

$$
[\overline{\mathbf{J}}(\mathbf{r}, p)]^{\dagger}=\overline{\mathbf{J}}\left(\mathbf{r}, p^{*}\right), \quad[\breve{\mathbf{J}}(\mathbf{r}, p)]^{\dagger}=\breve{\mathbf{J}}\left(\mathbf{r}, p^{*}\right),
$$

as follows from inspection of Eq. (27) and (33). Hence, the commutators of $\overline{\mathbf{J}}$ and $\breve{\mathbf{J}}$ can be written down immediately by using Eqs. (B1) and (B3). Subsequently, these commutation relations can be employed in evaluating the commutator of $\mathbf{J}(\mathbf{r}, \omega)$ with $\mathbf{J}\left(\mathbf{r}^{\prime}, \omega^{\prime}\right)$. We encounter terms with various 
numbers of Green-function factors. The contribution without a Green function is found to vanish:

$$
\begin{aligned}
& {\left[\overline{\mathbf{J}}(\mathbf{r},-i \omega+0)+\breve{\mathbf{J}}(\mathbf{r}, i \omega+0), \overline{\mathbf{J}}\left(\mathbf{r}^{\prime},-i \omega^{\prime}+0\right)+\breve{\mathbf{J}}\left(\mathbf{r}^{\prime}, i \omega^{\prime}+0\right)\right]} \\
& \quad=0 .
\end{aligned}
$$

In an analogous way as above, the terms with one and with two Green-function factors in the commutator $\left[\mathbf{J}(\mathbf{r}, \omega), \mathbf{J}\left(\mathbf{r}^{\prime}, \omega^{\prime}\right)\right]$ can be shown to cancel. This completes the proof of Eq. (65).

\section{APPENDIX C: EVALUATION OF THE HAMILTONIAN}

In order to show how the Hamiltonian (3) is recovered from Eq. (70), we give a few examples of the calculations that are involved. We shall demonstrate how the terms quadratic in $\boldsymbol{\Pi}$ and in $\mathbf{A}$ in Eq. (3) are obtained after substitution of Eq. (52) with (53a) and (53b) in Eq. (70). In this Appendix all canonical variables are taken at $t=0$.

We start with the term quadratic in II. After substitution of the appropriate expressions we get the following contribution to the Hamiltonian:

$$
\frac{1}{\pi \varepsilon_{0} c^{4}} \int d \mathbf{r} \int_{0}^{\infty} d \omega \omega^{3} \chi_{i}(\mathbf{r}, \omega) \int d \mathbf{r}^{\prime} \int d \mathbf{r}^{\prime \prime} \boldsymbol{\Pi}\left(\mathbf{r}^{\prime}\right) \cdot \overline{\mathbf{G}}\left(\mathbf{r}^{\prime}, \mathbf{r},-i \omega+0\right) \cdot \overline{\mathbf{G}}\left(\mathbf{r}, \mathbf{r}^{\prime \prime}, i \omega+0\right) \cdot \boldsymbol{\Pi}\left(\mathbf{r}^{\prime \prime}\right) .
$$

We could replace the transverse parts of the Green functions by the full Green functions, as $\boldsymbol{\Pi}$ is purely transverse. Let us now rewrite $\chi_{i}(\mathbf{r}, \omega)$ as $-(i / 2)[\bar{\chi}(\mathbf{r},-i \omega+0)-\bar{\chi}(\mathbf{r}, i \omega+0)]$. Subsequently, we carry out the integral over $\mathbf{r}$ by means of the optical theorem, which follows from Eq. (B7) by taking $\omega^{\prime}=\omega$. In doing so, the integral with the spatial derivatives in Eq. (B7) drops out, whereas the contributions with a single Green function in Eq. (B7) remain. In this way we find from Eq. (C1):

$$
\begin{gathered}
-\frac{i}{2 \pi \varepsilon_{0} c^{2}} \int_{0}^{\infty} d \omega \omega \int d \mathbf{r}^{\prime} \int d \mathbf{r}^{\prime \prime} \Pi\left(\mathbf{r}^{\prime}\right) \cdot\left[\overline{\mathbf{G}}\left(\mathbf{r}^{\prime}, \mathbf{r}^{\prime \prime}, i \omega+0\right)\right. \\
\left.-\overline{\mathbf{G}}\left(\mathbf{r}^{\prime}, \mathbf{r}^{\prime \prime},-i \omega+0\right)\right] \cdot \boldsymbol{\Pi}\left(\mathbf{r}^{\prime \prime}\right) .
\end{gathered}
$$

The integral over the frequency can now be performed with the use of the sum rule Eq. (A4). We finally obtain the simple result $\left(2 \varepsilon_{0}\right)^{-1} \int d \mathbf{r}[\Pi(\mathbf{I})]^{2}$, as in Eq. (3).

As a second example, we consider the terms quadratic in A. From Eqs. (70) with (52) and (53a) we get a similar expression as in Eq. (C1). The main difference is a factor $\omega^{5}$ instead of $\omega^{3}$ in the integrand. After performing the integral over $\mathbf{r}$ as before, we arrive at

$$
\begin{aligned}
& -\frac{i \varepsilon_{0}}{2 \pi c^{2}} \int_{0}^{\infty} d \omega \omega^{3} \int d \mathbf{r}^{\prime} \int d \mathbf{r}^{\prime \prime} \mathbf{A}\left(\mathbf{r}^{\prime}\right) \cdot\left[\overline{\mathbf{G}}\left(\mathbf{r}^{\prime}, \mathbf{r}^{\prime \prime}, i \omega+0\right)\right. \\
& \left.-\overline{\mathbf{G}}\left(\mathbf{r}^{\prime}, \mathbf{r}^{\prime \prime},-i \omega+0\right)\right] \cdot \mathbf{A}\left(\mathbf{r}^{\prime \prime}\right) .
\end{aligned}
$$

Owing to the presence of the factor $\omega^{3}$ we cannot use the sum rule Eq. (A4). However, we may proceed by adding and subtracting the asymptotic form Eq. (A3). In this way, the $\omega$-integral becomes:

$$
-\int_{-\infty}^{\infty} d \omega \omega^{3}\left[\overline{\mathbf{G}}\left(\mathbf{r}, \mathbf{r}^{\prime},-i \omega+0\right)-\frac{c^{2}}{(\omega+i 0)^{2}} \mathbf{I} \delta\left(\mathbf{r}-\mathbf{r}^{\prime}\right)\right],
$$

where we relabeled the position variables. Since the asymptotic form of the susceptibility for large $\omega$ is $-\left[\alpha^{2} /\left(\varepsilon_{0} \rho\right)\right] /(\omega+i 0)^{2}$, as we have seen in Appendix A, one derives from the differential equation for the Green function the asymptotic form for large $\omega$ :

$$
\begin{gathered}
\overline{\mathbf{G}}\left(\mathbf{r}, \mathbf{r}^{\prime},-i \omega+0\right)-\frac{c^{2}}{(\omega+i 0)^{2}} \mathbf{I} \delta\left(\mathbf{r}-\mathbf{r}^{\prime}\right) \\
\simeq \frac{c^{4}}{(\omega+i 0)^{4}}\left[(\boldsymbol{\nabla} \boldsymbol{\nabla}-\mathbf{I} \Delta) \delta\left(\mathbf{r}-\mathbf{r}^{\prime}\right)\right. \\
\left.+\frac{\mu_{0} \alpha^{2}}{\rho} \mathbf{I} \delta\left(\mathbf{r}-\mathbf{r}^{\prime}\right)\right],
\end{gathered}
$$

as a generalization of Eq. (A3). Employing this asymptotic form in Eq. (C4) one finds by contour integration the sum rule:

$$
\begin{aligned}
& \int_{-\infty}^{\infty} d \omega \omega^{3}\left[\overline{\mathbf{G}}\left(\mathbf{r}, \mathbf{r}^{\prime},-i \omega+0\right)-\frac{c^{2}}{(\omega+i 0)^{2}} \mathbf{I} \delta\left(\mathbf{r}-\mathbf{r}^{\prime}\right)\right] \\
&=-i \pi c^{4}\left[(\boldsymbol{\nabla} \nabla-\mathbf{I} \Delta) \delta\left(\mathbf{r}-\mathbf{r}^{\prime}\right)+\frac{\mu_{0} \alpha^{2}}{\rho} \mathbf{I} \delta\left(\mathbf{r}-\mathbf{r}^{\prime}\right)\right] .
\end{aligned}
$$

Substitution in Eq. (C3) yields

$$
\begin{gathered}
\frac{1}{2 \mu_{0}} \int d \mathbf{r} \mathbf{A}(\mathbf{r}) \cdot(\boldsymbol{\nabla} \nabla-\mathbf{I} \Delta) \cdot \mathbf{A}(\mathbf{r})+\int d \mathbf{r} \frac{\alpha^{2}}{2 \rho}[\mathbf{A}(\mathbf{r})]^{2} \\
=\frac{1}{2 \mu_{0}} \int d \mathbf{r}[\boldsymbol{\nabla} \times \mathbf{A}(\mathbf{r})]^{2}+\int d \mathbf{r} \frac{\alpha^{2}}{2 \rho}[\mathbf{A}(\mathbf{r})]^{2},
\end{gathered}
$$

which agrees with the contributions in Eq. (3).

Similar techniques can be used to obtain the other terms in the Hamiltonian (3). Several additional sum rules, with integrands containing products of the Green function and the susceptibility, are needed in establishing complete agreement. These are: 


$$
\begin{gathered}
\int_{-\infty}^{\infty} d \omega \omega^{3} \bar{\chi}(\mathbf{r},-i \omega+0) \overline{\mathbf{G}}\left(\mathbf{r}, \mathbf{r}^{\prime},-i \omega+0\right) \bar{\chi}\left(\mathbf{r}^{\prime},-i \omega+0\right)=0, \\
\int_{-\infty}^{\infty} d \omega \omega^{5} \bar{\chi}(\mathbf{r},-i \omega+0) \overline{\mathbf{G}}\left(\mathbf{r}, \mathbf{r}^{\prime},-i \omega+0\right) \bar{\chi}\left(\mathbf{r}^{\prime},-i \omega+0\right) \\
=-\frac{i \pi c^{2} \alpha^{4}}{\varepsilon_{0}^{2} \rho^{2}} \mathbf{I} \delta\left(\mathbf{r}-\mathbf{r}^{\prime}\right) .
\end{gathered}
$$

$$
\begin{aligned}
\mathbf{c}_{A X}\left(\mathbf{r}, \mathbf{r}^{\prime}, \omega\right)= & -\frac{i \mu_{0} \alpha^{\prime}}{\pi} \operatorname{Im}\left[\mathbf{G}_{\mathrm{TT}^{\prime}}\left(\mathbf{r}, \mathbf{r}^{\prime}, \omega\right)\right] \\
& -\frac{i \rho^{\prime}}{\pi c^{2} \alpha^{\prime}} \omega^{2} \operatorname{Im}\left[\mathbf{G}_{\mathrm{T}}\left(\mathbf{r}, \mathbf{r}^{\prime}, \omega\right) \chi\left(\mathbf{r}^{\prime}, \omega\right)\right]
\end{aligned}
$$

$$
\mathbf{c}_{A P}\left(\mathbf{r}, \mathbf{r}^{\prime}, \omega\right)=\frac{1}{\pi c^{2} \alpha^{\prime}} \omega \operatorname{Im}\left[\mathbf{G}_{\mathrm{T}}\left(\mathbf{r}, \mathbf{r}^{\prime}, \omega\right) \chi\left(\mathbf{r}^{\prime}, \omega\right)\right]
$$

Furthermore, one needs two sum rules for the susceptibility:

$$
\begin{gathered}
\int_{-\infty}^{\infty} d \omega \omega \bar{\chi}(\mathbf{r},-i \omega+0)=\frac{i \pi \alpha^{2}}{\varepsilon_{0} \rho}, \\
\int_{-\infty}^{\infty} d \omega \omega^{3}\left[\bar{\chi}(\mathbf{r},-i \omega+0)+\frac{\alpha^{2}}{\varepsilon_{0} \rho} \frac{1}{(\omega+i 0)^{2}}\right]=\frac{i \pi \alpha^{2} \widetilde{\omega}_{0}^{2}}{\varepsilon_{0} \rho} .
\end{gathered}
$$

To prove these sum rules one uses a contour deformation and the asymptotic behavior of the integrands, as before.

\section{APPENDIX D: TIME-DEPENDENT OPERATORS}

In this Appendix we show how time-dependent operators can be expressed as linear combinations of the canonical variables. As examples we shall discuss the vector potential, the electric field, and the polarization density.

The time-dependent vector potential has been given in Eq. (71). By substituting the expression (52) for the noise-current density, and employing the same methods as used in checking Eq. (68) we derive

$$
\begin{aligned}
\mathbf{A}(\mathbf{r}, t)= & \int d \mathbf{r}^{\prime} \int_{0}^{\infty} d \omega e^{-i \omega t}\left\{\mathbf{c}_{A A}\left(\mathbf{r}, \mathbf{r}^{\prime}, \omega\right) \cdot \mathbf{A}\left(\mathbf{r}^{\prime}, 0\right)\right. \\
& +\mathbf{c}_{A \Pi}\left(\mathbf{r}, \mathbf{r}^{\prime}, \omega\right) \cdot \boldsymbol{\Pi}\left(\mathbf{r}^{\prime}, 0\right)+\mathbf{c}_{A X}\left(\mathbf{r}, \mathbf{r}^{\prime}, \omega\right) \cdot \mathbf{X}\left(\mathbf{r}^{\prime}, 0\right) \\
& +\mathbf{C}_{A P}\left(\mathbf{r}, \mathbf{r}^{\prime}, \omega\right) \cdot \mathbf{P}\left(\mathbf{r}^{\prime}, 0\right) \\
& +\int_{0}^{\infty} d \omega^{\prime} \mathbf{C}_{A Y Q}\left(\mathbf{r}, \mathbf{r}^{\prime}, \omega, \omega^{\prime}\right) \cdot\left[\omega^{\prime 2} \mathbf{Y}_{\omega^{\prime}}\left(\mathbf{r}^{\prime}, 0\right)\right. \\
& \left.\left.+\frac{i \omega}{\rho^{\prime}} \mathbf{Q}_{\omega^{\prime}}\left(\mathbf{r}^{\prime}, 0\right)\right]\right\}+ \text { H.c. }
\end{aligned}
$$

The coefficients are

$$
\begin{gathered}
\mathbf{c}_{A A}\left(\mathbf{r}, \mathbf{r}^{\prime}, \omega\right)=-\frac{1}{\pi c^{2}} \omega \operatorname{Im}\left[\mathbf{G}_{\mathrm{TT}^{\prime}}\left(\mathbf{r}, \mathbf{r}^{\prime}, \omega\right)\right] \\
\mathbf{c}_{A \Pi}\left(\mathbf{r}, \mathbf{r}^{\prime}, \omega\right)=-\frac{i \mu_{0}}{\pi} \operatorname{Im}\left[\mathbf{G}_{\mathrm{TT}}\left(\mathbf{r}, \mathbf{r}^{\prime}, \omega\right)\right]
\end{gathered}
$$

$$
\begin{aligned}
\mathbf{c}_{A Y Q}\left(\mathbf{r}, \mathbf{r}^{\prime}, \omega, \omega^{\prime}\right)= & \frac{1}{\pi c^{2} \alpha^{\prime}} \operatorname{Im}\left[\frac{\omega v_{\omega^{\prime}}^{\prime}}{\omega^{\prime 2}-(\omega+i 0)^{2}}\right. \\
& \left.\times \mathbf{G}_{\mathrm{T}}\left(\mathbf{r}, \mathbf{r}^{\prime}, \omega\right) \chi\left(\mathbf{r}^{\prime}, \omega\right)\right] .
\end{aligned}
$$

The expression (D1) gives the vector potential $\mathbf{A}(\mathbf{r}, t)$ for all $t$ in terms of the canonical variables at $t=0$. In particular, it may be used to determine the vector potential for large $t$, when all transients have died out. In order to derive this asymptotic form, one starts by noting that the term with the Hermitian conjugate in (D1) can be used to extend the $\omega$-integral over the whole real axis. For all positive $t$ this integral may be evaluated by deforming the contour in the lower half-plane. If $t$ gets large, the behavior of $\mathbf{A}(\mathbf{r}, t)$ is dominated by the contributions from those singularities of the frequency-dependent integrand that are located close to the real $\omega$-axis in the lower half-plane. To find these singularities we consider the contributions from the various terms in Eq. (D1) one by one. The contributions involving $\mathbf{A}, \boldsymbol{\Pi}$, $\mathbf{X}$, and $\mathbf{P}$ depend on the coefficients (D2a)-(D2c). The singularities in these coefficients are determined by those of (the analytical continuations of) $\chi(\mathbf{r}, \omega)$ and $\mathbf{G}\left(\mathbf{r}, \mathbf{r}^{\prime}, \omega\right)$. The Green function $\mathbf{G}_{\mathrm{TT}^{\prime}}$ in Eqs. (D2a)-(D2c) is defined as the convolution of the Green function $\mathbf{G}$ with two transverse delta functions, one at its left and the other at its right-hand side. When the susceptibility has a finite imaginary part for real $\omega$, continuity implies that the singularities of $\chi$ and $\mathbf{G}$ in the lower half-plane are located at a finite distance from the real $\omega$-axis. As a consequence, the contributions from the terms with $\mathbf{A}, \boldsymbol{\Pi}, \mathbf{X}$, and $\mathbf{P}$ in Eq. (D1) will die out exponentially fast for large $t$. On the other hand, some of the singularities of the coefficient (D2e) are really close to the real axis, as they are given by $\omega= \pm \omega^{\prime}-i 0$. The contributions from these singularities will dominate the behavior of Eq. (D1) for large $t$. These contributions are readily evaluated by calculating the residues. One arrives at the result given in Eq. (72) of the main text. It shows that the long-time behavior of $\mathbf{A}(\mathbf{r}, t)$ is governed by the specific combination $\mathbf{J}_{l}(\mathbf{r}, \omega)$ of bath operators, given in Eq. (73):

$$
\begin{aligned}
\mathbf{A}(\mathbf{r}, t) \simeq & -\mu_{0} \int d \mathbf{r}^{\prime} \int_{0}^{\infty} d \omega e^{-i \omega t} \mathbf{G}_{\mathrm{T}}\left(\mathbf{r}, \mathbf{r}^{\prime}, \omega\right) \cdot \mathbf{J}_{l}\left(\mathbf{r}^{\prime}, \omega\right) \\
& + \text { H.c. }
\end{aligned}
$$

As noted in the main text, the combination of bath operators 
occurring in $\mathbf{J}_{l}(\mathbf{r}, \omega)$ is in fact proportional to the annihilation operator of the bath harmonic oscillator at $\mathbf{r}$ and with frequency $\omega$.

The time-dependent electric field $\mathbf{E}(\mathbf{r}, t)$ can be evaluated in an analogous way. Substituting Eq. (52) in (74) we obtain an expression like Eq. (D1). The coefficients $\mathbf{c}_{E i}$ (with $i$ $=A, \Pi, X, P, Y Q$ ) follow from $\mathbf{c}_{A i}$ in Eq. (D2) upon multiplying each coefficient by $i \omega$ and dropping the subscript $\mathrm{T}$ (but not $\mathrm{T}^{\prime}$ ) of the Green functions. The analysis of the long-time behavior of the electric field is completely analogous to that of the vector potential. One finds an expression of the same form as Eq. (74), with $\mathbf{J}$ replaced by $\mathbf{J}_{l}$ :

$$
\begin{aligned}
\mathbf{E}(\mathbf{r}, t) \simeq & -i \mu_{0} \int d \mathbf{r}^{\prime} \int_{0}^{\infty} d \omega e^{-i \omega t} \omega \mathbf{G}\left(\mathbf{r}, \mathbf{r}^{\prime}, \omega\right) \cdot \mathbf{J}_{l}\left(\mathbf{r}^{\prime}, \omega\right) \\
& + \text { H.c. }
\end{aligned}
$$

Finally, we consider the time-dependent polarization density. From Eqs. (75) and (52) with (53) we derive an expression for $\mathbf{X}(\mathbf{r}, t)$, which has the same form as Eq. (D1), with the coefficients:

$$
\mathbf{c}_{X A}\left(\mathbf{r}, \mathbf{r}^{\prime}, \omega\right)=\frac{i \varepsilon_{0}}{\pi c^{2} \alpha} \omega^{2} \operatorname{Im}\left[\chi(\mathbf{r}, \omega) \mathbf{G}_{\mathrm{T}^{\prime}}\left(\mathbf{r}, \mathbf{r}^{\prime}, \omega\right)\right]
$$

$$
\mathbf{c}_{X \Pi}\left(\mathbf{r}, \mathbf{r}^{\prime}, \omega\right)=-\frac{1}{\pi c^{2} \alpha} \omega \operatorname{Im}\left[\chi(\mathbf{r}, \omega) \mathbf{G}_{\mathrm{T}^{\prime}}\left(\mathbf{r}, \mathbf{r}^{\prime}, \omega\right)\right]
$$

$$
\begin{aligned}
\mathbf{c}_{X X}\left(\mathbf{r}, \mathbf{r}^{\prime}, \omega\right)= & \frac{\varepsilon_{0} \rho}{\pi \alpha^{2}} \omega \chi_{i}(\mathbf{r}, \omega) \mathbf{I} \delta\left(\mathbf{r}-\mathbf{r}^{\prime}\right) \\
& -\frac{\alpha^{\prime}}{\pi c^{2} \alpha} \omega \operatorname{Im}\left[\chi(\mathbf{r}, \omega) \mathbf{G}_{\mathrm{T}^{\prime}}\left(\mathbf{r}, \mathbf{r}^{\prime}, \omega\right)\right] \\
& -\frac{\varepsilon_{0} \rho^{\prime}}{\pi c^{2} \alpha \alpha^{\prime}} \omega^{3} \operatorname{Im}\left[\chi(\mathbf{r}, \omega) \mathbf{G}\left(\mathbf{r}, \mathbf{r}^{\prime}, \omega\right) \chi\left(\mathbf{r}^{\prime}, \omega\right)\right],
\end{aligned}
$$

$$
\begin{gathered}
\mathbf{c}_{X P}\left(\mathbf{r}, \mathbf{r}^{\prime}, \omega\right)= \\
\quad \frac{i \varepsilon_{0}}{\pi \alpha^{2}} \chi_{i}(\mathbf{r}, \omega) \mathbf{I} \delta\left(\mathbf{r}-\mathbf{r}^{\prime}\right) \\
\quad-\frac{i \varepsilon_{0}}{\pi c^{2} \alpha \alpha^{\prime}} \omega^{2} \operatorname{Im}\left[\chi(\mathbf{r}, \omega) \mathbf{G}\left(\mathbf{r}, \mathbf{r}^{\prime}, \omega\right) \chi\left(\mathbf{r}^{\prime}, \omega\right)\right], \\
\mathbf{c}_{X Y Q}\left(\mathbf{r}, \mathbf{r}^{\prime}, \omega, \omega^{\prime}\right) \\
=\frac{i \varepsilon_{0}}{\pi \alpha^{2}} \operatorname{Im}\left[\frac{v_{\omega^{\prime}}}{\omega^{\prime 2}-(\omega+i 0)^{2}} \chi(\mathbf{r}, \omega)\right] \mathbf{I} \delta\left(\mathbf{r}-\mathbf{r}^{\prime}\right) \\
-\frac{i \varepsilon_{0}}{\pi c^{2} \alpha \alpha^{\prime}} \operatorname{Im}\left[\frac{\omega^{2} v_{\omega^{\prime}}^{\prime}}{\omega^{\prime 2}-(\omega+i 0)^{2}}\right. \\
\left.\quad \times \chi(\mathbf{r}, \omega) \mathbf{G}\left(\mathbf{r}, \mathbf{r}^{\prime}, \omega\right) \chi\left(\mathbf{r}^{\prime}, \omega\right)\right] .
\end{gathered}
$$

The behavior in the long-time limit is dominated by the singularities close to the real frequency axis. These singularities arise from the coefficient in Eq. (D5e). Evaluating their contributions we arrive at the following expression for $\mathbf{X}(\mathbf{r}, t)$ in the long-time limit:

$$
\begin{aligned}
\mathbf{X}(\mathbf{r}, t) \simeq & \frac{i}{c^{2} \alpha} \int d \mathbf{r}^{\prime} \int_{0}^{\infty} d \omega e^{-i \omega t} \omega \chi(\mathbf{r}, \omega) \\
& \times \mathbf{G}\left(\mathbf{r}, \mathbf{r}^{\prime}, \omega\right) \cdot \mathbf{J}_{l}\left(\mathbf{r}^{\prime}, \omega\right) \\
& -\frac{i}{\alpha} \int_{0}^{\infty} d \omega e^{-i \omega t} \frac{1}{\omega} \mathbf{J}_{l}(\mathbf{r}, \omega)+\text { H.c. },
\end{aligned}
$$

with $\mathbf{J}_{l}$ given by Eq. (73). Upon multiplying with $-\alpha$ and comparing with Eq. (76), we see that the long-time limit has the same effect on the polarization density as it has on the vector potential and the electric field: the dependence on the full noise-current density $\mathbf{J}(\mathbf{r}, \omega)$ is replaced by a dependence on $\mathbf{J}_{l}(\mathbf{r}, \omega)$.
[1] J. M. Jauch and K. M. Watson, Phys. Rev. 74, 950 (1948); 74, 1485 (1948).

[2] L. Knöll, W. Vogel, and D.-G. Welsch, Phys. Rev. A 36, 3803 (1987).

[3] R. J. Glauber and M. Lewenstein, Phys. Rev. A 43, 467 (1991).

[4] L. Knöll and D.-G. Welsch, Prog. Quantum Electron. 16, 135 (1992).

[5] B. J. Dalton, E. S. Guerra, and P. L. Knight, Phys. Rev. A 54, 2292 (1996).

[6] B. J. Dalton and M. Babiker, Phys. Rev. A 56, 905 (1997).

[7] M. Wubs, L. G. Suttorp, and A. Lagendijk, Phys. Rev. A 68, 013822 (2003).

[8] B. Huttner and S. M. Barnett, Europhys. Lett. 18, 487 (1992).

[9] B. Huttner and S. M. Barnett, Phys. Rev. A 46, 4306 (1992).
[10] J. J. Hopfield, Phys. Rev. 112, 1555 (1958).

[11] S. M. Barnett, B. Huttner, and R. Loudon, Phys. Rev. Lett. 68, 3698 (1992).

[12] S. M. Dutra and K. Furuya, Europhys. Lett. 43, 13 (1998).

[13] S. M. Dutra and K. Furuya, Phys. Rev. A 57, 3050 (1998).

[14] A. Bechler, J. Mod. Opt. 46, 901 (1999).

[15] M. Wubs and L. G. Suttorp, Phys. Rev. A 63, 043809 (2001).

[16] M. Janowicz, D. Reddig, and M. Holthaus, Phys. Rev. A 68 , 043823 (2003).

[17] T. Gruner and D.-G. Welsch, Phys. Rev. A 51, 3246 (1995).

[18] R. Matloob, R. Loudon, S. M. Barnett, and J. Jeffers, Phys. Rev. A 52, 4823 (1995).

[19] T. Gruner and D.-G. Welsch, Phys. Rev. A 53, 1818 (1996).

[20] R. Matloob and R. Loudon, Phys. Rev. A 53, 4567 (1996).

[21] T. Gruner and D.-G. Welsch, Phys. Rev. A 54, 1661 (1996). 
[22] H. T. Dung, L. Knöll, and D.-G. Welsch, Phys. Rev. A 57, 3931 (1998).

[23] S. Scheel, L. Knöll, and D.-G. Welsch, Phys. Rev. A 58, 700 (1998).

[24] A. Tip, Phys. Rev. A 57, 4818 (1998).

[25] A. Tip, L. Knöll, S. Scheel, and D. G. Welsch, Phys. Rev. A 63, 043806 (2001).

[26] L. Knöll, S. Scheel, and D.-G. Welsch, in Coherence and Statistics of Photons and Atoms, edited by J. Peřina (Wiley, New York, 2001), p. 1.

[27] A. Lukš and V. Peřinová, in Progress in Optics, edited by E. Wolf (North-Holland, Amsterdam, 2002), Vol. 43, p. 295.

[28] S. M. Barnett, B. Huttner, R. Loudon, and R. Matloob, J. Phys. B 29, 3763 (1996).

[29] S. Scheel, L. Knöll, D.-G. Welsch, and S. M. Barnett, Phys. Rev. A 60, 1590 (1999).

[30] S. Scheel, L. Knöll, and D.-G. Welsch, Phys. Rev. A 60, 4094 (1999); 61, 069901 (2000) [errata].

[31] H. T. Dung, L. Knöll, and D.-G. Welsch, Phys. Rev. A 62,
053804 (2000).

[32] H. T. Dung, L. Knöll, and D.-G. Welsch, Phys. Rev. A 65, 043813 (2002).

[33] H. T. Dung, L. Knöll, and D.-G. Welsch, Phys. Rev. A 66, 063810 (2002)

[34] S. M. Barnett, C. R. Gilson, B. Huttner, and N. Imoto, Phys. Rev. Lett. 77, 1739 (1996).

[35] S. M. Barnett, J. Jeffers, A. Gatti, and R. Loudon, Phys. Rev. A 57, 2134 (1998).

[36] O. Di Stefano, S. Savasta, and R. Girlanda, Phys. Rev. A 61, 023803 (2000).

[37] M. Artoni and R. Loudon, Phys. Rev. A 59, 2279 (1999).

[38] S. Scheel, L. Knöll, T. Opatrný, and D.-G. Welsch, Phys. Rev. A 62, 043803 (2000).

[39] M. S. Yeung and T. K. Gustafson, Phys. Rev. A 54, 5227 (1996).

[40] U. Fano, Phys. Rev. 124, 1866 (1961).

[41] A. J. van Wonderen and L. G. Suttorp, Europhys. Lett. (to be published). 